Article

\title{
Estimating Crop Albedo in the Application of a Physical Model Based on the Law of Energy Conservation and Spectral Invariants
}

\author{
Jingjing Peng ${ }^{1,2}$, Wenjie Fan ${ }^{1,3, *}$, Xiru Xu ${ }^{1,3, \dagger}$, Lizhao Wang ${ }^{4, \dagger}$, Qinhuo Liu ${ }^{2,5, *}$, Jveai Li ${ }^{1,3}$ \\ and Peng Zhao ${ }^{1,3}$
}

1 Institute of RS and GIS, Peking University, Beijing 100871, China; E-Mails: rspeggy@ 163.com (J.P.); xrxurs@126.com (X.X.); $1401210311 @$ pku.edu.cn (J.L.); zhaopengowen@126.com (P.Z.)

2 State Key Laboratory of Remote Sensing Science, jointly sponsored by Institute of Remote Sensing and Digital Earth of CAS and Beijing Normal University, Beijing 100101, China;

3 The Beijing Key Laboratory of Spatial Information Integration and 3S Application, Beijing 100871, China

4 Beijing Research Institute of Automation for Machinery Industry, Beijing 100120, China; E-Mail: wlz1220@sina.com

5 Joint Center for Global Change Studies, Beijing 100875, China

$\dagger$ These authors contributed equally to this work.

* Authors to whom correspondence should be addressed; E-Mails: fanwj@pku.edu.cn (W.F.); liuqh@radi.ac.cn (Q.L.); Tel.: +86-010-6275-5085 (W.F.).

Academic Editors: Xin Li, Yuei-An Liou, Richard Müller and Prasad S. Thenkabail

Received: 4 August 2015 / Accepted: 10 November 2015 / Published: 18 November 2015

\begin{abstract}
Albedo characterizes the radiometric interface of land surfaces, especially vegetation, and the atmosphere. Albedo is a critical input to many models, such as crop growth models, hydrological models and climate models. For the extensive attention to crop monitoring, a physical albedo model for crops is developed based on the law of energy conservation and spectral invariants, which is derived from a prior forest albedo model. The model inputs have been efficiently and physically parameterized, including the dependency of albedo on the solar zenith/azimuth angle, the fraction of diffuse skylight in the incident radiance, the canopy structure, the leaf reflectance/transmittance and the soil reflectance characteristics. Both the anisotropy of soil reflectance and the clumping effect of crop leaves at the canopy scale are considered, which contribute to the improvement of the model accuracy. The comparison between the model results and Monte Carlo
\end{abstract}


simulation results indicates that the canopy albedo has high accuracy with an RMSE $<0.005$. The validation using ground measurements has also demonstrated the reliability of the model and that it can reflect the interaction mechanism between radiation and the canopy-soil system.

Keywords: albedo; crop; spectral invariant

\section{Introduction}

Surface albedo determines the shortwave radiation budget and the energy transfers at the soil-vegetation-atmosphere interface and further influences of ecosystem, hydrology and meteorology processes, such as photosynthesis and evaporation [1]. As vegetation plays a crucial role in all of these natural processes, carefully depicting the vegetation albedo is of prime interest when studying crop monitoring [2], hydrological cycle modeling [3,4], weather forecasting and climate modeling [5]. Compared to other vegetation types, this crop requires extra attention, because it shows significant seasonal variation and strong fluctuations caused by human activities. Its spectral characteristics change along with its growth as the proportion of bare soil decreases. Currently, a great number of research studies are beginning to focus on the modeling and inversion of crop characteristics, especially the crop albedo $[5,6]$.

Previously, the albedo of a certain land cover type was set to a constant in many application cases [7]. Along with the development of quantitative remote sensing (QRS) techniques, increasing near real-time satellite albedo data have provided the opportunity to develop advanced land surface albedo parameterization [8,9], such as the wide application of MODIS albedo products [9].

Albedo is usually calculated as the weighted average of black-sky albedo (BSA) and white-sky albedo (WSA). BSA is the angular integration of the bidirectional reflectance distribution function (BRDF) or bidirectional reflectance factor (BRF) over all viewing directions, whereas WSA is a further integration of BSA over all illumination directions [10]. The vegetation albedo varies intricately due to the abundant variation of the vegetation bidirectional reflectance distribution function (BRDF) [8]. The vegetation BRDF modeling involves two physical processes: surface scattering and volume scattering. They are best handled by geometric optic (GO) theory and radiative transfer (RT) theory, respectively [11]. However, direct integration based on these models to obtain vegetation albedo is too complex to formulate mathematically [12].

To solve this problem, a semi-empirical kernel-driven model based on the linear combination of isotropic, geometric and volume scattering kernels has been developed $[13,14]$. The model has greatly simplified the integration process. However, the model hides the land cover and crop structural information in the statistical kernel coefficients, which cannot be directly used in crop monitoring [15]. Furthermore, the inversion of the model relies on atmospherically-corrected, cloud-cleared, multi-angular and multi-date reflectance observations [13]. Thus, the temporal resolution of albedo is hard to improve, and the model is not suitable for high spatial resolution data without prior knowledge [16].

As albedo is also defined as the ratio of upwelling flux (irradiance) to downward flux (irradiance) [10], it can be estimated without integration by accumulating the upward and downward irradiances using 
numerical simulation methods [17]. The downward flux, i.e., the sum of the direct solar flux and the diffuse solar flux, which is an integration of the sky radiance over all illumination directions, varies with solar zenith angle and atmospheric visibility [10]. The upward flux depends on not only the downward flux, but also the land surface characteristics. A common method used for calculation of the radiation reflected by a canopy is the Monte Carle (MC) method, by which the intensity of the reflected radiation can be obtained if a sufficient number of photons is traced [18,19]. Accurate as the MC simulation is, it requires a complete description of the scene, and the radiation is obtained by counting without knowing the mathematical relationships between the parameters.

The " $p$-theory" starts by depicting the manner in which photons interact with the canopy and understory [20]. The recollision probability $(p)$ is spectrally invariant and can be interpreted as the probability that a photon scattered from a leaf will interact ("collide") with another leaf in the canopy, which is used to predict the amount of radiation scattered by the canopy [21]. Based on the $p$-theory, Manninen and Stenberg [22] estimated the vegetation-ground (forest floor) multiple scattering and simulated the forest albedo. However, the $p$-theory is effective by assuming that the scatter phases of leaves are isotropous, that is that the derivative models cannot describe the angular distribution of the scattered radiation in regard to the collision of photons with leaves. This causes some discrepancy from the realistic situation, which can be neglected on average at large scales [23]. As the calculation of the absorbed radiation is more succinct than that of the reflected radiation, the albedo was calculated as the complement of the canopy and soil absorption from the law of energy conservation by Stenberg [24].

The model of Stenberg [24] physically expresses the relationships between forest albedo and the canopy and understory parameters. The model was derived for a given direction of illumination (solar zenith angle). Commonly, ignoring the contribution of diffuse skylight may cause a $0.1 \%-2 \%$ estimation error of canopy albedo [25]. Therefore, to apply the model in real sceneries, an angular integration is necessary, although it would increase the calculation complexity. Fan et al. [26] parameterized the composition of the incident radiation using the fraction of diffuse irradiance in calculating canopy absorptance, which performs well for both continuous and non-continuous vegetation. Besides, in regard to the crop, the anisotropic reflectance characteristics of the soil should also be included, which dominate the IFOV before the closing of the crop canopy.

This paper aims to estimate the crop albedo in the base of the forest albedo model by Manninen et al. [22] and Stenberg [24]. Considering the characteristics of crop canopy, the clumping index of crop canopy was calculated to facilitate the model for non-continuous crop canopy, and the composition of incident radiation was parameterized. Besides, the Hapke model was used to calculate the directional-hemispheric reflectance and bi-hemispheric soil reflectance, both of which have been used in the crop albedo model to depict the anisotropic reflectance of the soil [27].

According to the sensitivity analysis of the main inputs, the model is simplified. The process was also checked and verified using Monte Carlo simulation. The final model was validated using field measurements.

\section{Model Description}

Surface albedo is determined by the surface properties and the angular and spectral distributions of the incident solar radiation. Vegetation canopy albedo depends on the leaf area index (LAI), leaf angle 
distribution, leaf transmittance, leaf reflectance and understory albedo, which is mainly influenced by the soil moisture of the crop [8]. The model is supposed to make explicit the relationships between these parameters and the vegetation canopy albedo.

Continuous vegetation is the simplest structural type for modeling. This section first introduces the model for a continuous canopy and then uses the clumping index to extend the model to a non-continuous crop. Finally, the model was simplified, so the dominant parameters and mechanisms are clearer and more distinct.

\subsection{Basic Model}

The model originates from the albedo model by Manninen and Stenberg [22], further decomposed by Stenberg et al. [24]. To adapt to the crop scenery, the model was further refined, which assumes the actual incoming light as a combination of direct and diffuse radiation, calculates the clumping index of the crop to add in the model and separates soil reflectance into directional-hemispherical and bi-hemispherical reflectance to discriminate the essence of the direct and indirect collision. The derivation of the model also refers to the FAPAR model by Fan et al. [26].

According to the law of energy conservation, the albedo (A) of vegetation land cover is the complement of the canopy absorptance $a v(\lambda)$ and the soil absorptance $a v(\lambda)$, which can be described as,

$$
A(\lambda)=1-a v(\lambda)-a s(\lambda)
$$

The $a v(\lambda)$ includes the direct absorption of radiation by the canopy $a v_{1}(\lambda)$ and the part that is reflected by the background and then absorbed by the vegetation $a v_{2}(\lambda)$.

$$
a v(\lambda)=a v_{1}(\lambda)+a v_{2}(\lambda)
$$

The as $(\lambda)$ includes the fraction of solar radiation directly absorbed by the soil $a s_{1}(\lambda)$ and that caused by vegetation-soil multiple scattering $a s_{2}(\lambda)$ (Figure 1).

$$
a s(\lambda)=a s_{1}(\lambda)+a s_{2}(\lambda)
$$

The energy reaching the soil $\left(f^{\downarrow}\right)$ includes two parts, the proportion of the incident radiation that directly reaches the soil without interacting with the canopy $\left(f_{l}\right)$ and that after one or more scattering events within the canopy $\left(f_{2}\right) . f_{l}$ has a certain incidence angle, and the reflected radiation is determined by the directional-hemispheric reflectance of the soil $\left(r_{g}^{d}\right)$. Moreover, the reflected radiation from the proportion that reaches the soil after one or more scattering events within the canopy $\left(f_{h}\right)$ is influenced by the bi-hemispherical reflectance of the soil $\left(r_{g}^{h}\right)$.

$$
\begin{gathered}
a s_{1}=f_{1} \cdot\left(1-r_{g}^{d}\left(\theta_{s}\right)\right) \\
a s_{2}(\lambda)=f_{h}(\lambda) \cdot \frac{1-r_{g}^{h}}{1-r_{g}^{h} \tilde{i_{0}} r_{c}^{*}} \\
r_{c}^{*}=\frac{\omega_{l} q_{2}}{1-p \omega_{l}}
\end{gathered}
$$


where $r_{c}^{*}$ is the diffuse reflectance of the vegetation canopy. $q_{2}$ is the fraction of incoming radiation scattered downwards (i.e., forward). $\tilde{i}_{0}$ is the average interception probability of the diffuse skylight by the canopy, which can be interpreted as the angular integration of the directional canopy interception of the incoming radiation $i_{0}$ [22].

The canopy interception $i_{0}$ is expressed as $i_{0}=1-e^{-\frac{\Omega \cdot G_{\theta} \cdot L A I}{\cos \theta}}$ based on the Poisson law. $\tilde{i}_{0}$ is integrated from the directional interception probability $i_{0}$ [22].

$$
\tilde{i_{0}}=\frac{1}{\pi} \int_{2 \pi}\left(1-e^{-\frac{\Omega \cdot G_{\theta} \cdot L A I}{\cos \theta}}\right) \cos \theta \sin \theta d \theta d \varphi
$$

In practice, $\tilde{i}_{0}$ can be obtained from numerical integration. Fan et al. [26] proposed an empirical exponential function of $\tilde{i}_{0}$ for the spherical vegetation type.

$f_{1}$ depends on the canopy transmittance, as well as the composition of the incident radiation. $\beta$ discriminates the contribution of the direct sunlight and the diffuse skylight.

$$
f_{1}=(1-\beta) \cdot\left(1-i_{0}\right)+\beta\left(1-\tilde{i}_{0}\right)
$$

$f_{h}(\lambda)$ is the sum of two parts, the reflected direct sunlight after the first collision with the soil $\left(f_{1} r_{g}^{d} \tilde{i}_{0} r_{c}^{*}\right)$ and the proportion that reaches the soil after one or more interception by the canopy through scattering $\left(f_{2}\right)$.

$$
f_{h}(\lambda)=f_{1} r_{g}^{d} \tilde{i}_{0} r_{c}^{*}+f_{2}
$$

$f_{2}$ also depends on the factor of the diffuse skylight and the directional/diffuse interception by the vegetation canopy through scattering.

$$
f_{2}=\left(i_{0} \cdot(1-\beta)+\tilde{i}_{0} \cdot \beta\right) \cdot r_{c}^{*}
$$

Then, a physical model of vegetation albedo has been formed as follows (symbols listed in Table 1),

$$
\begin{aligned}
& A(\lambda)=1-a v(\lambda)-a s(\lambda) \\
& \alpha v(\lambda)=\left[\left(i_{0} \cdot(1-\beta)+\tilde{i}_{0} \cdot \beta\right)+f^{\downarrow} \cdot \frac{r_{g}^{h}}{1-r_{g}^{h} \tilde{i_{0}} r_{c}^{*}} \cdot \tilde{i_{0}}\right] \cdot \frac{1-\omega_{l}(\lambda)}{1-p \omega_{l}(\lambda)} \\
& \alpha s(\lambda)=\left[\begin{array}{l}
{\left[(1-\beta) \cdot\left(1-i_{0}\right)+\beta\left(1-\tilde{i}_{0}\right)\right] \cdot\left(1-r_{g}^{d}\right)} \\
+\left[(1-\beta) i_{0}+\tilde{i}_{0} \beta\right] \cdot \frac{r_{c}^{*}}{1-r_{g}^{h} \cdot \tilde{i}_{0} \cdot r_{c}^{*}} \cdot\left(1-r_{g}^{h}\right) \\
+\left[(1-\beta) \cdot\left(1-i_{0}\right)+\beta\left(1-\tilde{i}_{0}\right)\right] \cdot \frac{r_{g}^{d} \cdot \tilde{i}_{0} \cdot r_{c}^{*}}{1-r_{g}^{h} \cdot \tilde{i}_{0} \cdot r_{c}^{*}} \cdot\left(1-r_{g}^{h}\right)
\end{array}\right]
\end{aligned}
$$




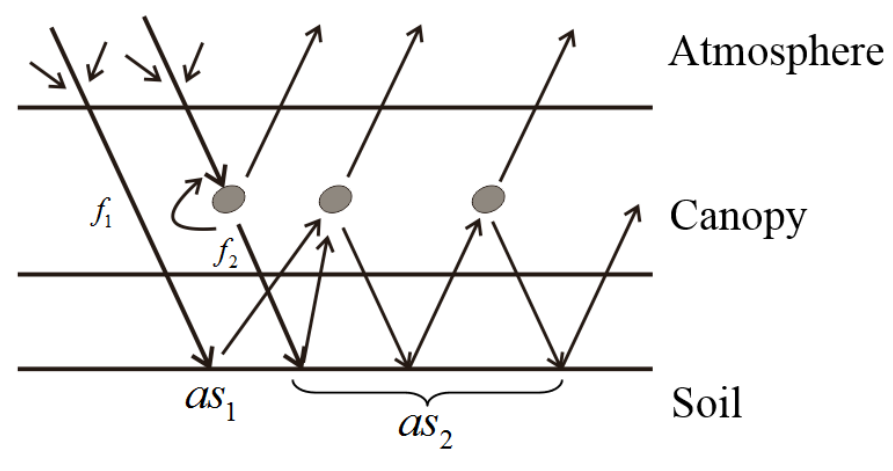

Figure 1. Illustration of the composition of soil absorption.

Table 1. Explanations of the symbols in the albedo model (the last four lines list the unknown parameters in the Hapke model).

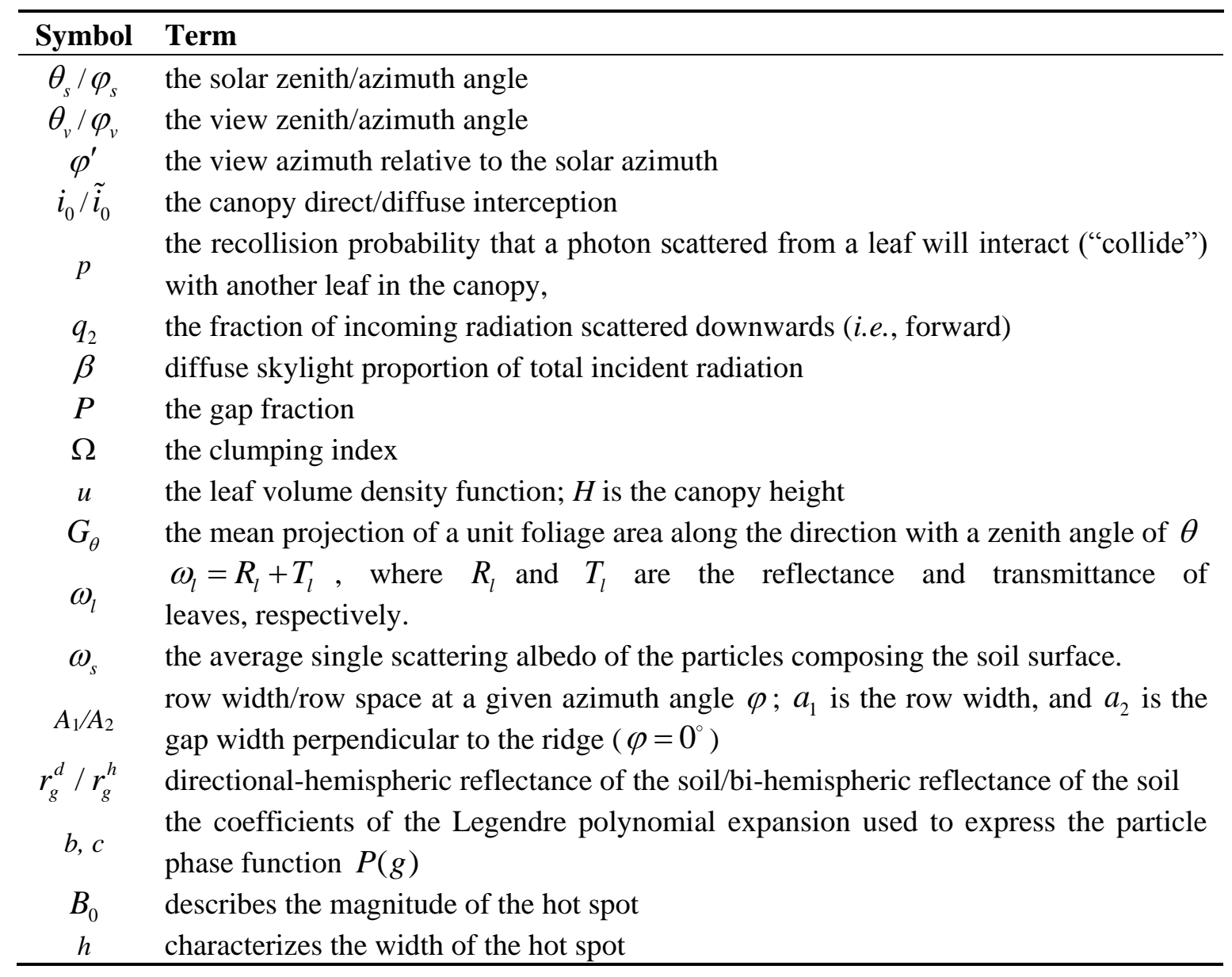

\subsection{For Noncontinuous Vegetation}

\subsubsection{Clumping Effect}

There are two groups of parameters determined by the canopy structure, which is affected by the clumping degree of the canopy. One is the canopy interception $i_{0}, \tilde{i}_{0}$ and transmittance $t_{0}$, and the other is the recollision probability $p$. With an assumption of continuous homogeneous vegetation under ideal conditions, these parameters can be directly calculated from the leaf area index (LAI) [26,28]. 
However, in nature, canopy transmittance increases in some directions, because leaves clump within crowns and among crowns [29]. The clumping effect has been considered in the calculation of the $p$ parameter by Stenberg [28].

The clumping index $\Omega(0 \leq \Omega \leq 1)$ is able to describe the clumping effect [30]. For non-continuous or non-uniform vegetation, the average gap fraction can be derived with the assistance of the clumping index [31].

$$
\overline{P(\theta, \varphi)}=\exp \left(-\Omega \frac{G_{\theta}}{\mu} L A I_{s}\right)
$$

Here, $L A I_{s}$ is an equivalent element area index determined by both the leaves and the stalks, which can be transformed to effective $L A I\left(L A I_{e}\right)$ by multiplying $\Omega$. In reverse, the clumping index can be retrieved from the average gap fraction given the direction $(\mu=\cos (\theta)), L A I_{s}$ and $\mathrm{G}$.

$$
\Omega=-\frac{\mu \ln (\overline{P(\theta, \varphi)})}{G_{\theta} L A I_{s}}
$$

The actual crop is always planted in rows (ridges). The clumping effect of crop is mainly caused at the canopy scale, i.e., the regular configuration between the rows. Each row is abstracted as a rectangular porous solid stretch along the ridge direction [33] (Figure 2).

In the calculation of $\Omega, L A I_{s}$ is difficult to quantify, because the contribution of crop stalks at different zenith angles is hardly measurable. Here, we adopted an approximation method. For randomly-distributed canopy structures, the distribution can be described using the Poisson theory. Meanwhile, for non-continuous vegetation with patches at various scales, the Neyman-type distribution fits more accurately, which assumes canopy first combined in groups, and the spatial distribution of the center of a group follows the Poisson process [32]. There is a parameter $\mathrm{m}_{2}$ in the Neyman distribution function that corresponds to the a priori value of the mean size of clusters. As the $\mathrm{m}_{2}$ is smaller, the Neyman distribution and Poisson distribution become closer. Assuming leaves distribute randomly within each row, it can be inferred that the crop will behave like continuous vegetation when the $\theta_{v}$ is larger than a threshold zenith angle $\left(\theta_{c}=\operatorname{atan}(A / H)\right)$, as the soil is invisible, which results in the clumping index levels going to an asymptote $(\approx 1)$. Therefore, the $L A I_{s}$ can be approximated to spatially-averaged leaf area index $L A I_{a}$ in calculating the average gap fraction. Therefore, the clumping index can be approximately calculated from Equation (14), which is also proven by Yan et al. [33],

$$
\Omega \cong-\frac{\mu \ln (\overline{P(\theta, \varphi)})}{G_{\theta} L A I_{a}}
$$

Yan et al. [33] derived the average gap fraction with a high accuracy; however, an integral method was misused in the calculation. Instead, the canopy should be segmented into many micro-slices, whose gap fraction add up to the final result. 


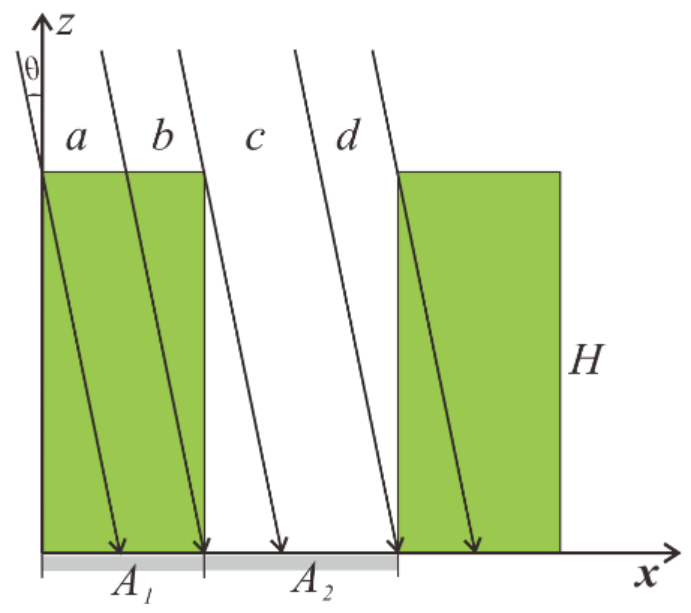

Figure 2. Illustration of average gap fraction calculation of row crops in a vertical plane with $x$ points to an azimuth angle of $\varphi$ (the azimuth increases in the clockwise direction with its origin along the row direction). $A=A_{1}+A_{2}$.

A row unit $A$ can be divided into four parts, namely $a, b, c$ and $d$. Part $a$ can be treated as continuous canopy. Parts $b$ and $d$ have equal gap fractions. They have been segmented into micro-slices, and each micro-slice can be considered as continuous canopy, as long as it is thin enough. The gap fraction of the four parts can be calculated as follows,

$$
\begin{aligned}
& \overline{P_{a}}=\exp \left(-\Omega \frac{G_{\theta}}{\cos \theta} u H\right), \Omega=1 ; \quad \overline{P_{c}}=1, \\
& \overline{P_{b}}=\overline{P_{d}}=\frac{\sum_{i=1}^{n} e^{-\frac{G_{\theta} \cdot u \cdot \cdot \cdot \Delta x}{\sin \theta}}}{H \cdot \tan \theta}=\frac{\cos \theta}{G_{\theta} \cdot u \cdot H}\left[1-e^{-\frac{G_{\theta} \cdot u \cdot H}{\cos \theta}}\right], \\
& n \cdot \Delta x=H \cdot \tan \theta
\end{aligned}
$$

where $A_{1}=a_{1} / \sin \varphi, A_{2}=a_{2} / \sin \varphi$ and $a_{1}$ and $a_{2}$ refer to the row width and row space, respectively, when the $x$-axis is perpendicular to the ridge $\left(\varphi=90^{\circ}\right)$. The average gap fraction of a crop unit is the weighted average of the four parts.

$$
\begin{aligned}
& \overline{P(\theta, \varphi)}=\frac{A_{1}-H \cdot \tan \theta}{A} \cdot \overline{P_{a}}+2 \cdot \frac{H \cdot \tan \theta}{A} \cdot \overline{P_{b}}+ \\
& \frac{A_{2}-H \cdot \tan \theta}{A} \cdot \overline{P_{c}}=\frac{1}{A}\left[\begin{array}{l}
\left(A_{1}-H \cdot \tan \theta-2 \cdot \frac{\sin \theta}{G_{\theta} \cdot u}\right) \cdot e^{-\frac{G_{\theta} \cdot u \cdot H}{\cos \theta}} \\
+\left(A_{2}-H \cdot \tan \theta+2 \cdot \frac{\sin \theta}{G_{\theta} \cdot u}\right)
\end{array}\right]
\end{aligned}
$$

\subsubsection{Anisotropic Soil Reflectance}

For continuous vegetation, the influence of soil anisotropy on canopy albedo is not as obvious as that for the crop, especially in the beginning of the growing season [34]. For this crop albedo model, the Hapke model was used to simulate the BRDF of soil and to build a look-up table of soil $r_{g}^{d}$ through 
angular integration. $r_{g}^{h}$ was further obtained through illumination hemisphere integration of $r_{g}^{d}$. The Hapke model is expressed as Equation (17); refer to [35] for detailed information.

$$
r\left(\theta_{s}, \theta_{v}, \varphi^{\prime}\right)=\frac{\omega_{s}}{4} \frac{1}{\cos \theta_{s}+\cos \theta_{v}}\left\{[1+B(g)] P(g)+H\left(\cos \theta_{s}\right) H\left(\cos \theta_{v}\right)-1\right\}
$$

where

$$
\begin{aligned}
& B(g)=\frac{B_{0}}{[1+(1 / h) \tan (g / 2)]}, \\
& P(g)=1+b \cos g+c\left[\left(3 \cos ^{2} g-1\right) / 2\right], \\
& H(x)=\left\{\frac{(1+2 x)}{[1+2 \sqrt{(1-\omega)} x]}\right\} .
\end{aligned}
$$

\subsection{Model Simplification}

Sufficient detail, as Equation (11), shows that the entire model is slightly complicated and has increased inversion difficulties. In fact, the contributions of some components are really limited, so much as to be neglected. The model was simplified and reorganized as Equation (18). There are two simplified parts in $a v(\lambda)$, both of which relate to the absorption of soil-reflected radiation by the canopy. One is neglecting the difference between directional and hemispherical canopy interception $\left(i_{0}\right.$ and $\tilde{i}_{0}$ ) only before the first collision between photons and the soil surface. The other is omitting the factor $r_{g}^{h} \cdot \tilde{i}_{0} \cdot r_{c}^{*}$ in the denominator, as it is close to zero. Similarly to as $(\lambda)$, two simplifications were made. One is that the first part of $f_{h}(\lambda)$ (Equation (9)) is neglected, because the absorption from the multi-collision after the first direct collision between canopy and soil is too small. The difference between $r_{g}^{d}$ and $r_{g}^{h}$ is mainly embodied in the first direct collision and multi-indirect collision. Second is that the factor $r_{g}^{h} \cdot \tilde{i}_{0} \cdot r_{c}^{*}$ is omitted, the same as that in $a v(\lambda)$. The difference between the simplified model and the original model is simulated and illustrated in Figure 3.

$$
\begin{gathered}
A(\lambda)=1-a v(\lambda)-a s(\lambda) \\
a v(\lambda) \approx\left(i_{0}+\left(\tilde{i}_{0}-i_{0}\right) \cdot \beta+\left(1-i_{0}\right) \cdot r_{g}^{h} \cdot \tilde{i}_{0}\right) \cdot \frac{1-\omega_{l}(\lambda)}{1-p \omega_{l}(\lambda)} \\
\operatorname{as}(\lambda) \approx\left[\left(1-i_{0}\right)+\beta\left(i_{0}-\tilde{i}_{0}\right)\right] \cdot\left(1-r_{g}^{d}\right)+\left[i_{0}+\beta\left(\tilde{i}_{0}-i_{0}\right)\right] \cdot r_{c}^{*} \cdot\left(1-r_{g}^{h}\right)
\end{gathered}
$$

The simplified model gave a clear physical explanation of the vegetation albedo. There are three mutual factors for vegetation absorption and soil absorption: the vegetation interception, the factor of diffuse skylight and the soil reflectance characteristics. Moreover, the vegetation absorption is also influenced by the spectral absorption coefficient $\left(a_{c}(\lambda)=\frac{1-\omega_{l}}{1-\omega_{l} p}\right)$, which is determined by the recollision probability $p$ and the leaf single scattering albedo $\left(\omega_{l}\right)$. 

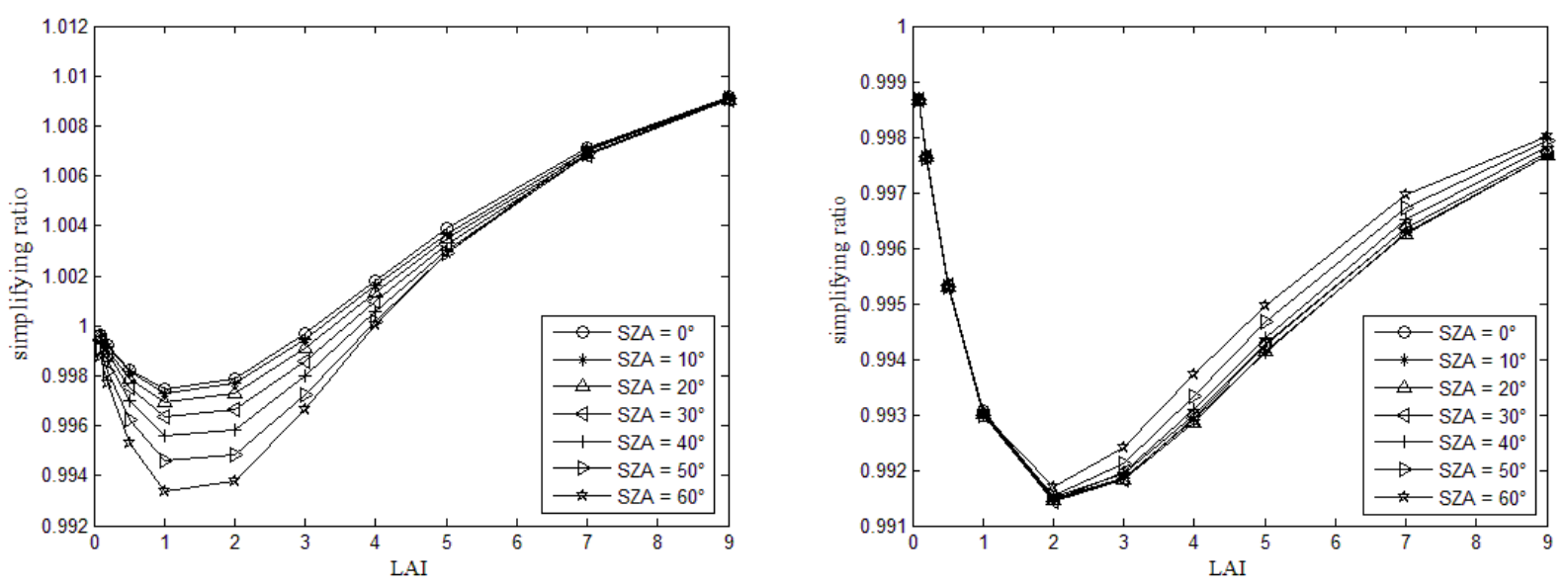

Figure 3. The ratio between the simplified value and the original value of $a v$ (left) and $a s$ (right). $\beta=0.1, \varphi=0^{\circ}, A_{1}=0.3, A_{2}=0.2, \omega_{l}=0.23$.

\section{Sensitivity and Scenario Analysis}

The factors discussed above, including the proportion of diffuse skylight, leaf area index and soil reflectance, contribute to the variation of vegetation albedo, as they influence canopy absorptance and soil absorptance. The sensitivity of albedo to these three parameters is analyzed in this section. The analysis of the first two parameters (Sections 3.1 and 3.2) was conducted on continuous vegetation, whereas the last one was conducted on crop, where the influence of soil reflectance is more significant. In the following simulations, $G_{\theta}$ were set as 0.5 . The directional-hemispherical reflectance $\left(r_{G}^{d}\right)$ of soil were simulated using the Hapke model. The five parameters of the Hapke model were set to: $\omega_{s}=0.54 ; B_{0}=0.33 ; h=0.21 ; b=0.86 ; c=0.70$.

\subsection{Variation of Albedo with the Diffuse Fraction}

In this algorithm, the vegetation albedo was derived from vegetation absorptance $a v(\lambda)$ and soil absorptance $a s(\lambda)$. For vegetation, the fraction of the incident diffuse radiation $\beta$ has an important impact on vegetation and soil absorptance. The sensitivity analysis was conducted on continuous vegetation. The simulation result in Figure 4 demonstrates that $a v(\lambda)$ is dominated by $a v_{1}(\lambda)$ and that $a s(\lambda)$ is dominated by $a s_{1}(\lambda)$. However, as the opposite effects of $\beta$ on $a v_{1}(\lambda)$ and $a s_{1}(\lambda)$ (referring to Equation (18)) offset each other, the albedo does not vary significantly with $\beta$. If the LAI is large enough and the reflectance of the leaves is smaller than that of the soil, the albedo would slightly increase with $\beta$, which explains the difference between vegetation albedo on cloudy days and sunny days.

\subsection{Variation of Vegetation Albedo along with LAI}

Along with the growth of LAI, the fraction of energy absorbed by the vegetation canopy increases, while the energy absorbed by soil decreases. However, because of the multiple interactions of photons at the soil-vegetation-atmosphere interface, the variation of albedo manifests in a complex manner. 
The influence of LAI on albedo is mainly determined by the canopy interception $\left(i_{0}\right.$ and $\left.\tilde{i}_{0}\right)$ and the recollision possibility $p$. The increase of LAI would trigger the growth of $i_{0}, \tilde{i}_{0}$ and $p$, and then $a v(\lambda)$ would increase and $a s(\lambda)$ decrease (Figure 5). Thus, the final variation of albedo results from the relative size of the leaf and soil reflectance. In Figure 5a, the canopy absorptance increases sharply as LAI grows, which is not adequately compensated by the decreasing of soil absorptance and leads to the decrease of canopy albedo. In Figure $5 \mathrm{~b}$, because $\omega_{s}$ is larger, the increasing of canopy absorptance is not as significant as the decrease of soil absorptance. Therefore, the canopy albedo slightly increases with the growth of LAI.

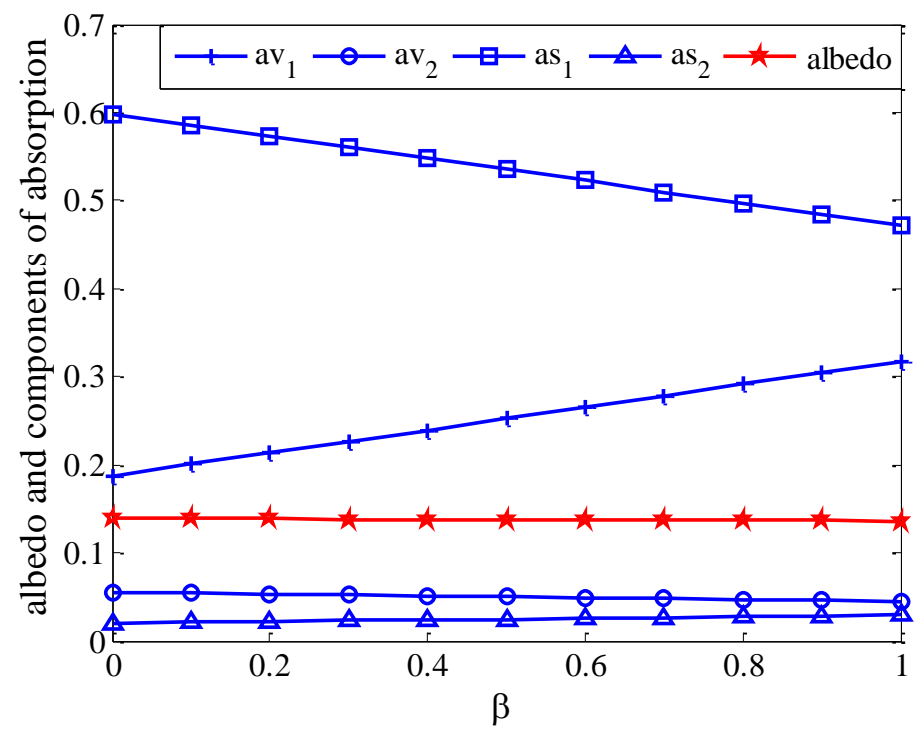

Figure 4. The determinants of the canopy albedo as a function of the fraction of diffuse radiation. The base values include $L A I=3, \theta_{s}=20^{\circ}$ and $\omega_{l}=0.23$ (at the green band). $\beta$ was varied from zero to one in steps of 0.1 , while other parameters were held constant.

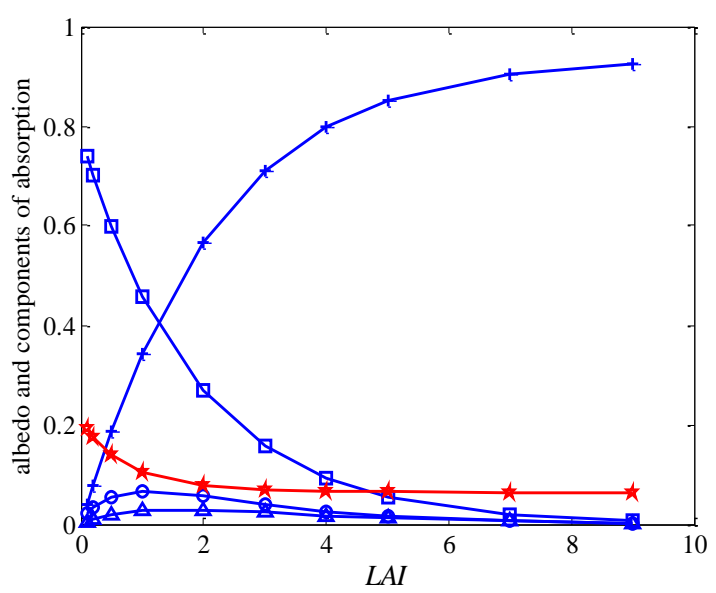

(a)

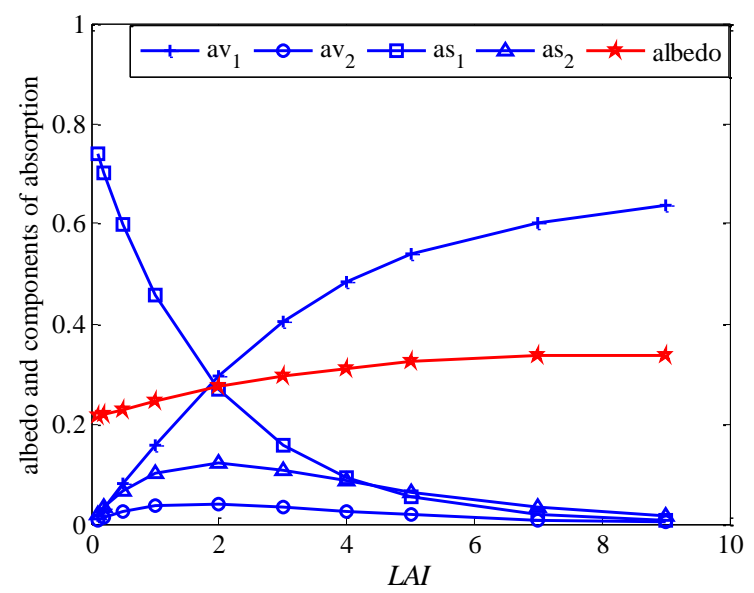

(b)

Figure 5. The determinants of the canopy albedo as functions of LAI: (a) $\omega_{l}=0.23$ (green band), $r_{g}^{h}=0.22, \theta_{s}=20^{\circ}$; (b) $\omega_{l}=0.7$ (near-infrared band), $r_{g}^{h}=0.22, \theta_{s}=20^{\circ}$. 


\subsection{Influence of Leaf Foliage Distribution on Canopy Albedo}

$G_{\theta}$ is the mean projection of a unit foliage area along the direction with a zenith angle of $\theta$, which is one of the parameters to describe canopy structure. There are five commonly-used G function types, including erectophile, plagiophile, spherical $(\mathrm{G}=0.5)$, extremophile and planophile [36]. In this model, the $\mathrm{G}$ function is employed to calculate the $i_{0}, \tilde{i}_{0}$ and $\overline{P(\theta, \varphi)}$. To clarify the impact of leaf foliage distribution on calculated albedo, a comparison was conducted between the albedo derived from these five $\mathrm{G}$ function types [36] with increasing LAI and other parameters constant.

Figure 6 manifests that the influence of G function is not apparent when LAI is extremely small or large (less than one or more than six) when $\omega_{l}=0.23, r_{g}^{h}=0.22, \theta_{s}=20^{\circ}$. The maximum discrepancy is about 0.006 when LAI is three.

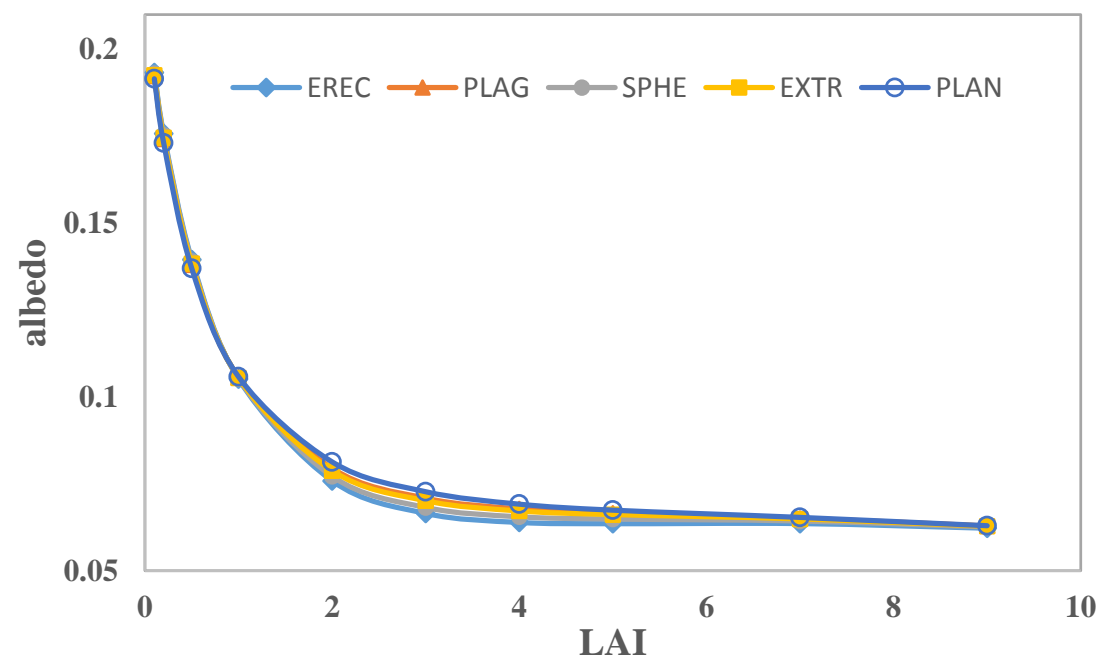

Figure 6. The canopy albedo derived from different $\mathrm{G}$ functions with changing LAI. (ERECerectophile, PLAG-plagiophile, SPHE-spherical, EXTR-extremophile and PLAN-planophile)

\subsection{Dependence of Canopy Albedo on Wavelength}

Albedo and canopy/soil absorption $(A(\lambda), a v(\lambda)$ and $a s(\lambda))$ change along with wavelength, owing to the spectrum-dependent parameters, including $\omega_{l}, r_{g}^{d}, r_{g}^{h}$ and $\beta$. Figure 7 shows the variation of $A(\lambda), a v(\lambda)$ and $a s(\lambda)$ derived by Equation (11), where spectral $\omega_{l}, r_{g}^{d}, r_{g}^{h}$ and $\beta$ are obtained from field measurements of maize crop. Figure 7 demonstrates that $a v(\lambda)$ and $a s(\lambda)$ appear to have the opposite variation trend and together determine the characteristics of $A(\lambda) . a v(\lambda)$ manifests a valley at the near-infrared band (760 $\mathrm{nm}$ to $1300 \mathrm{~nm}$ ) due to the structure of leaf cells. The less energy absorbed by the leaves, the more energy reaches the ground. Therefore, as $(\lambda)$ levels are maximum at the nearinfrared band. 


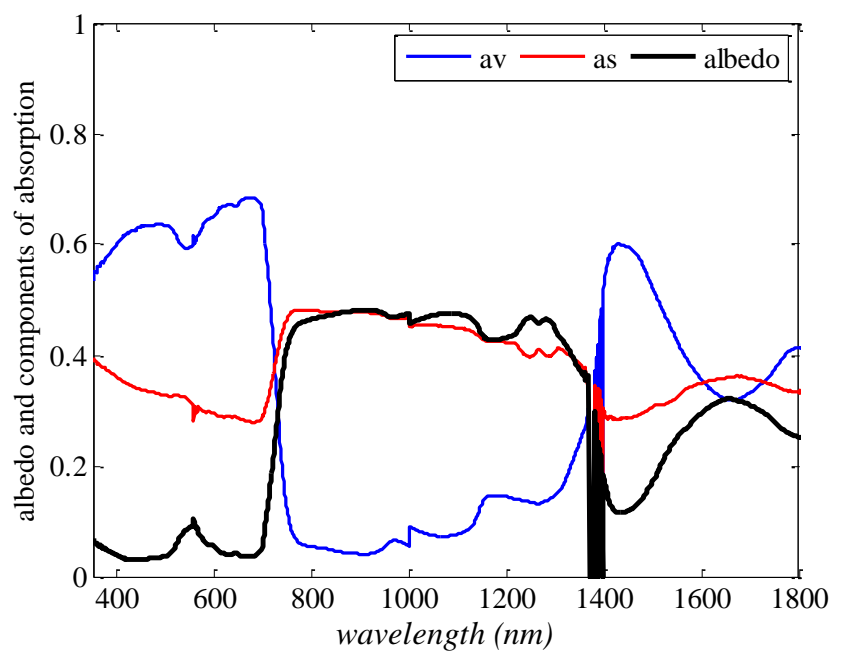

Figure 7. Spectral albedo and components of absorption.

\subsection{Influence of Soil Anisotropy on Canopy Albedo}

The albedo model discriminating the $r_{g}^{h}$ and $r_{g}^{d}$ is referred to as the anisotropy soil model, and the albedo model treating the soil as a Lambertian surface $\left(r_{g}^{h}\right)$ is called the isotropy soil model.

Figure 8 illustrates the discrepancy between the albedo derived from the isotropy soil model, which uses the soil $r_{g}^{d}$ as a constant, and that derived from the anisotropy soil model using varying $r_{g}^{d}$ as depicted in Figure 9. When the solar zenith angle is less than $60^{\circ}$, the albedo derived from the isotropy soil model is larger.

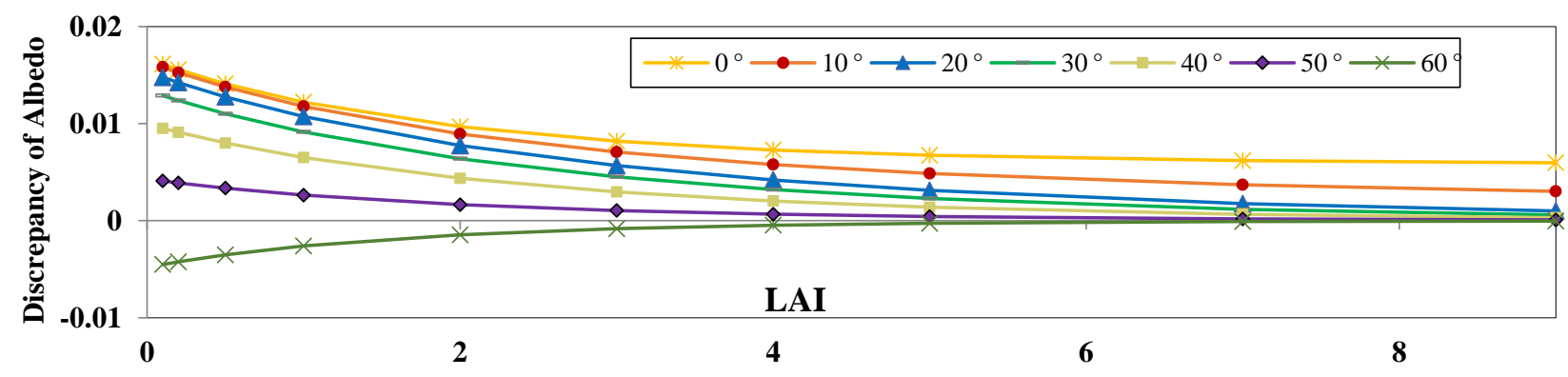

(a)

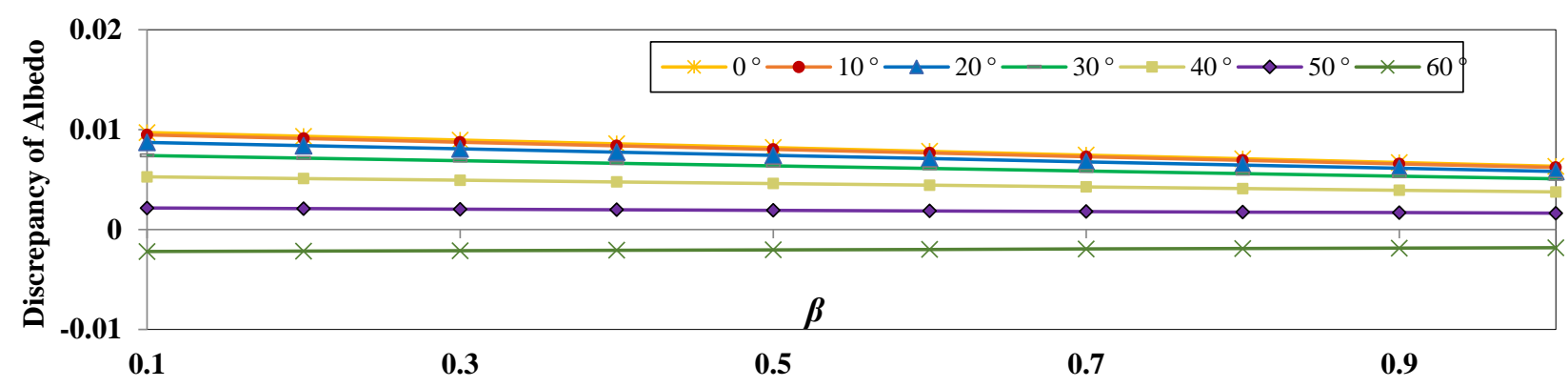

(b)

Figure 8. Cont. 


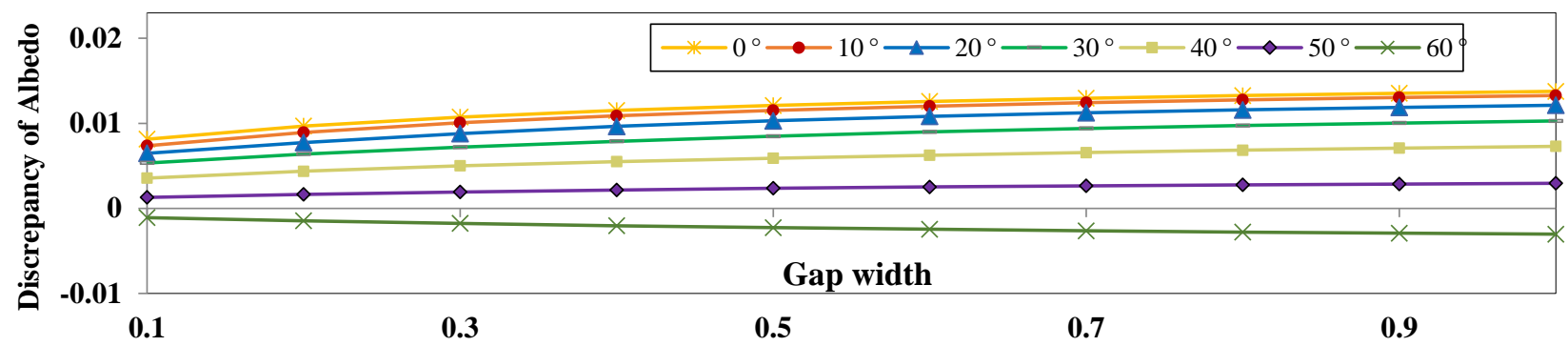

(c)

Figure 8. The discrepancy between albedo derived from the isotropy and anisotropy soil models. (a,b,c) the discrepancy of albedo varying with the LAI, $\beta$ and the gap width of crops. Each line corresponds to a certain $\theta_{s}$. The default values include $\omega_{l}=0.23, A_{1}=0.3$, $\mathrm{H}=1, \varphi=90^{\circ}$ (perpendicular to the ridge). (a) $\beta=0.2, A_{2}=0.2$, LAI was varied from 0.1 to nine. (b) $\mathrm{LAI}=2, A_{2}=0.2, \beta$ was varied from 0.1 to one. (c) $\mathrm{LAI}=2, \beta=0.2$, the gap width $\left(A_{2}\right)$ was varied from 0.1 to one.

According to Figure 9, this is because $r_{g}^{d}$ becomes larger than $r_{g}^{h}$ (which is equal to 0.2194 in this case) when the solar zenith angle is beyond $60^{\circ}$, and the albedo increases while $r_{g}^{d}$ increases, as Equation (17) explains.

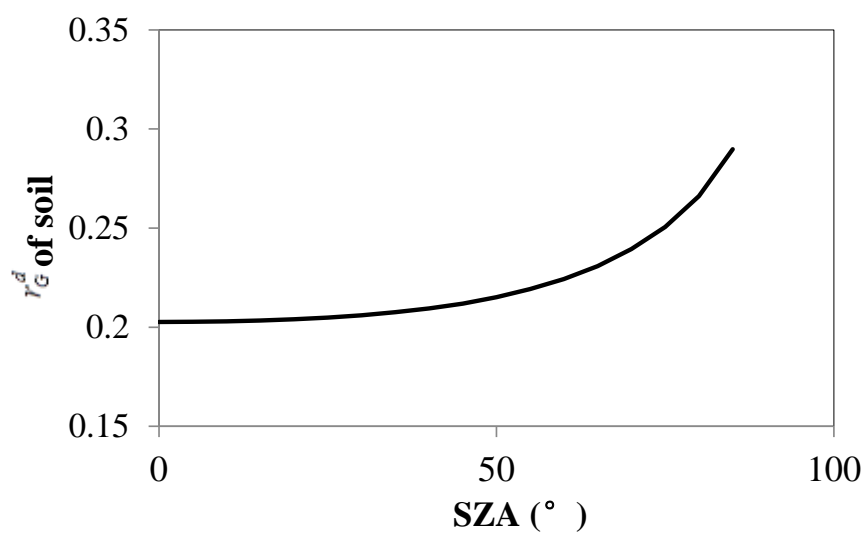

Figure 9. Variation of the directional-hemispherical reflectance $r_{g}^{d}$ along with the solar zenith angle (SZA).

In Figure 8a, the gap between the albedo at different SZA narrows, while the LAI increases, because soil is dominant in the field of view (FOV) at small LAI, whereas the fraction of vegetation in FOV increases, and the influence of soil reflectance decreases with the increase of LAI.

In Figure $8 \mathrm{~b}$, the gap between the albedo at different $\beta$ narrows with the increase of $\beta$. This is because the albedo derived from the isotropy soil model is barely changed with $\beta$, whereas the albedo derived from the anisotropy soil model grows with the increase of $\beta$.

In Figure 8c, the gap between the albedo widens with the increase of the gap width and a constant row width, because the dominancy of soil reflectance grows with the gap width.

In this case, the difference of albedo caused by the anisotropy of the soil is approximately \pm 0.02 , which needs to be considered when the accuracy demand is high. 


\section{Model Validation}

\subsection{Validation by MC Simulation}

\subsubsection{Method}

The model was validated using a Monte Carlo (MC) method. MC simulation is a numerical statistical method by which the radiative transfer process of photons in the canopy is simulated and the structural parameters of the canopy are predefined [18,26,37,38]. The main steps of the simulation include: generating the incident photons, determining the scenarios regarding photons colliding with leaves or soil, randomly assigning the scattering direction, calculating the free paths of the photons and counting the photons satisfying specific conditions.

The MC method was used to validate the albedo model under uniform continuous vegetation and crop conditions, respectively. White sky albedo (WSA) is the angular integration of black sky albedo (BSA) in the illumination hemisphere, so the BSA is mainly validated here. The diurnal variation of BSA depends on the solar zenith angle, and the long-term BSA mainly changes with effective LAI $\left(L A I_{e}\right)$. Therefore, the validation is conducted on two dimensions. One dimension is the LAI, and the other is the SZA. For uniform continuous vegetation, BSA remains constant with $\varphi_{s}$. Nevertheless, for the crop canopy, BSA would change with the variation of $\varphi_{s}$, as that would change the ratio between the crop width and canopy height. Therefore, two groups of perpendicular directions were selected with $\varphi_{s}$ values of $0^{\circ}$ and $90^{\circ}$, respectively. The parameters of the Hapke model were set as $\mathrm{w}=0.543$, $\mathrm{B}_{0}=0.33, \mathrm{~h}=0.21, \mathrm{~b}=0.86$ and $\mathrm{c}=0.70$, which were suitable for the soil characteristics of the measured soil in the research.

The main parameters involved include:

Leaf area index of the canopy: zero to nine;

Photon incident zenith angle $\left({ }^{\circ}\right)$ : zero to 60 ;

Photon incident azimuth angle $\left(^{\circ}\right)$ : zero, 90 ;

Leaf angle distribution type: spherical $(G=0.5)$;

Termination threshold of photon energy: 0.001 (controls when the round of simulation is terminated);

Photon number: $10^{6}$;

Soil directional-hemispherical reflectance $r_{g}^{d}$ : see Figure 9;

Soil bi-hemispherical reflectance $r_{g}^{h}: 0.2194$;

The leaf reflectance and leaf transmittance were obtained from the Leaf Optical Properties Experiment 93 (LOPEX93) database [26].

\subsubsection{Comparison of Continuous Vegetation}

The model-calculated black sky albedo (BSA) agrees well with the MC simulation result (Figure 10). The albedo decreases monotonically with LAI. A larger incident zenith angle corresponds to a higher albedo value.

The discrepancy between the two groups of results was evaluated in terms of Pearson's correlation when the leaf angular distribution type is spherical, the coefficient $(r)$ and the root mean square error 
(RMSE) (Figure 11). Results show that BSA simulated by MC and the model had a strong positive correlation $(r>0.999)$ and small discrepancy $(R M S E<0.003)$ under all solar zenith angles $<60^{\circ}$.

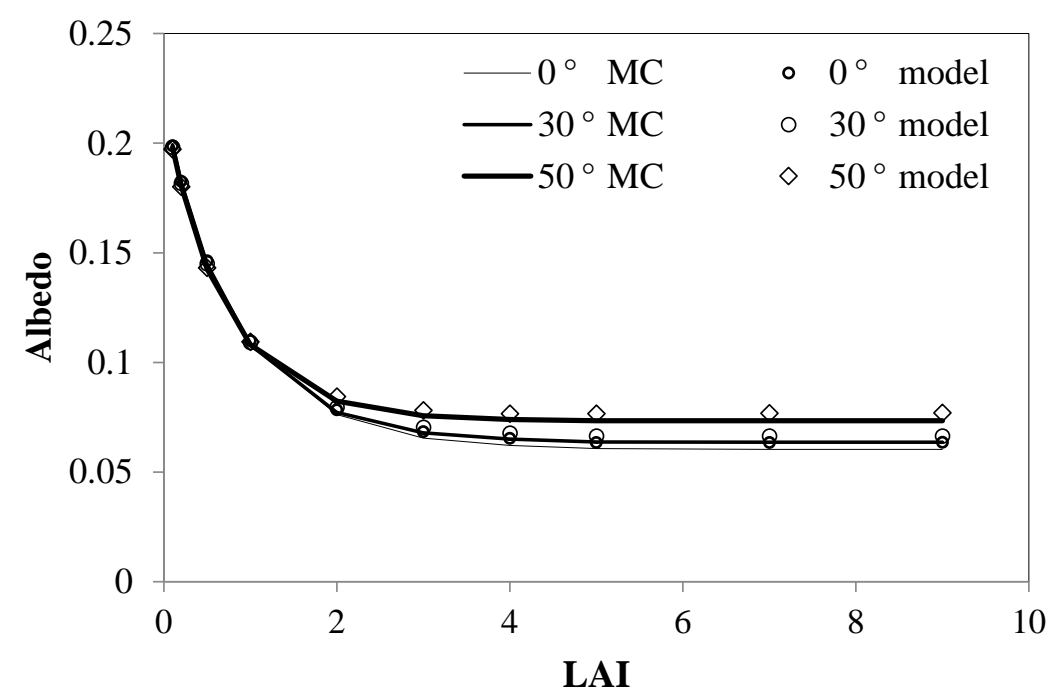

Figure 10. Relationships between LAI and black-sky albedo (BSA) as predicted by the model (circles and diamonds) and MC (solid lines) under different solar zenith angles (SZA, deployed at $0^{\circ}, 30^{\circ}$ and $50^{\circ}$ ) at the green band $(530 \mathrm{~nm})$. The difference in the width of lines and the size of markers shows the variation on SZA.

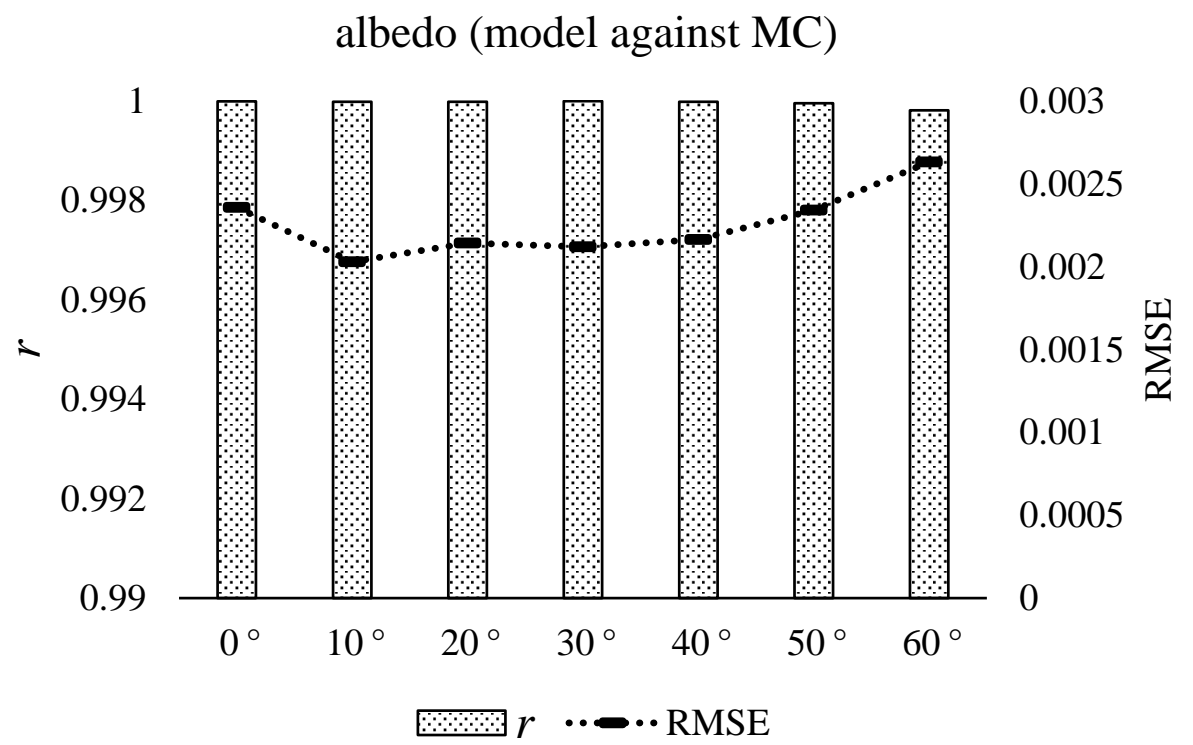

Figure 11. Pearson's correlation coefficient $(r)$ (bar graph) and root mean square error (RMSE) (line chart) between the model-simulated and MC-simulated BSA of continuous vegetation under different solar zenith angles at the green band $(530 \mathrm{~nm})$.

As well as the BSA, the $a v(\lambda)$ and $a s(\lambda)$ are of high accuracy according to the results illustrated in Figures 12 and 13. $a v(\lambda)$ increases while as $(\lambda)$ decreases monotonically with LAI and SZA. Both $a v(\lambda)$ and $a s(\lambda)$ derived from the model and from MC at different SZAs have strong agreements, with a high correlation $(r>0.999)$ and a small discrepancy $(R M S E<0.002)$. 


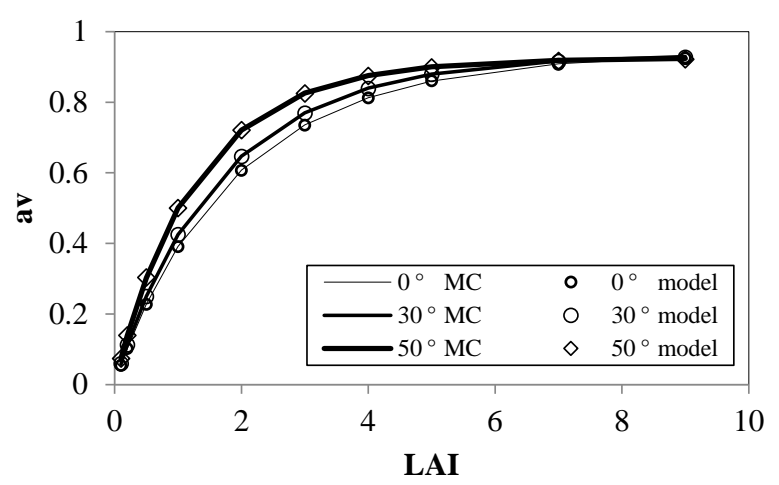

(a)

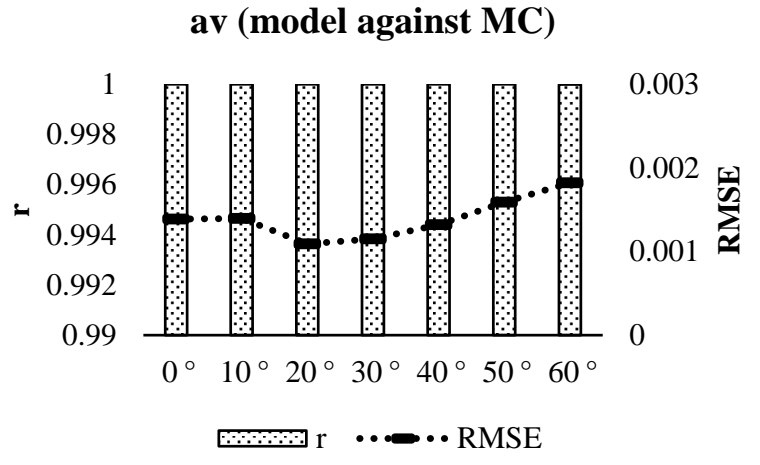

(b)

Figure 12. (a) Relationships between LAI and $a v$ as predicted by the model (circles and diamonds) and MC (lines) under different solar zenith angles at the green band; (b) the corresponding $r$ and RMSE.

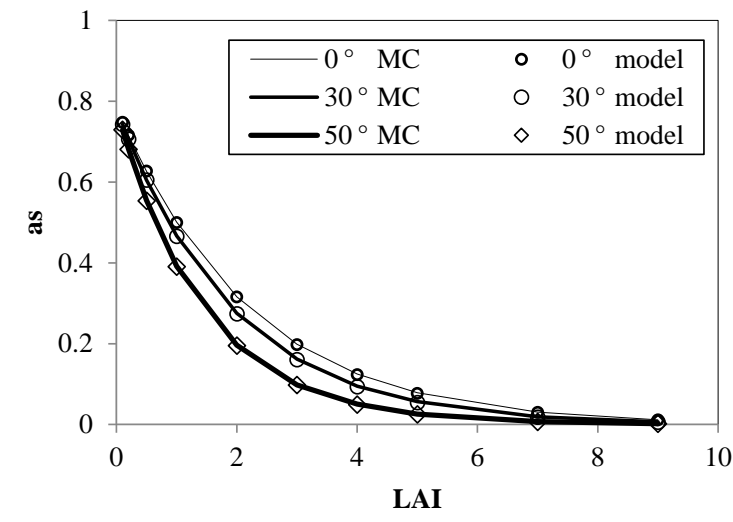

(a)

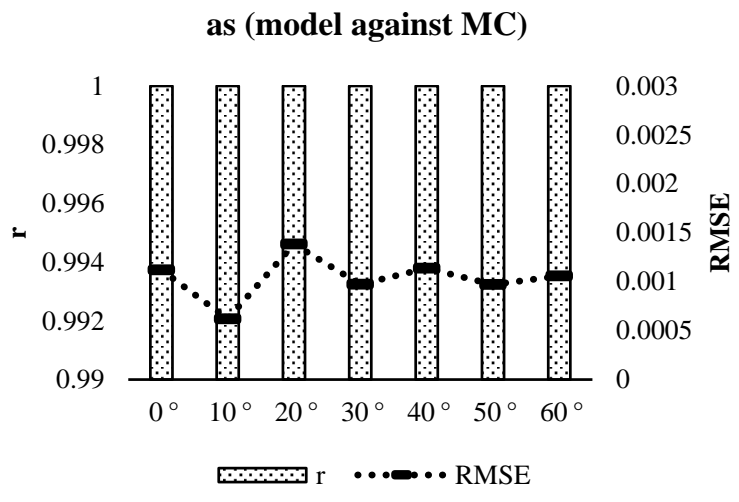

(b)

Figure 13. (a) Relationships between LAI and $a$ s as predicted by the model (circles and diamonds) and MC (lines) under different solar zenith angles at the green band; (b) the corresponding $r$ and RMSE.

\subsubsection{Validation for the Row Crop Canopy}

To understand the variation of simulation error under different LAI and incident zenith angles for the crop, the row width, gap width and canopy height were held constant at $0.3 \mathrm{~m}, 0.2 \mathrm{~m}$ and $1 \mathrm{~m}$, respectively, while LAI was varied from zero to nine and SZA varied from $0^{\circ}$ to $60^{\circ}$ in $10^{\circ}$ steps. Soil albedos were pre-simulated using the Hapke model. Figure 14 shows the validation results of the crop canopy when $\varphi=0^{\circ}$ (along the row direction) and $\varphi=90^{\circ}$ (perpendicular to the row). The high correlation coefficients and low RMSEs show that the values derived from the model are accurate for the crop canopy, as well as the continuous vegetation. 


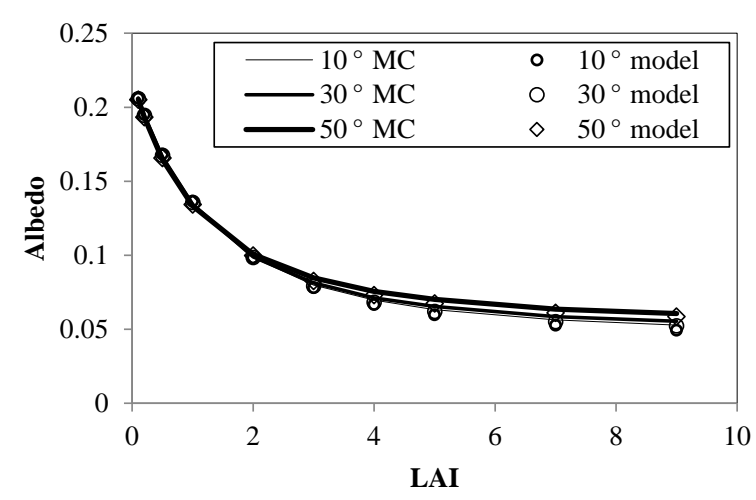

(a)

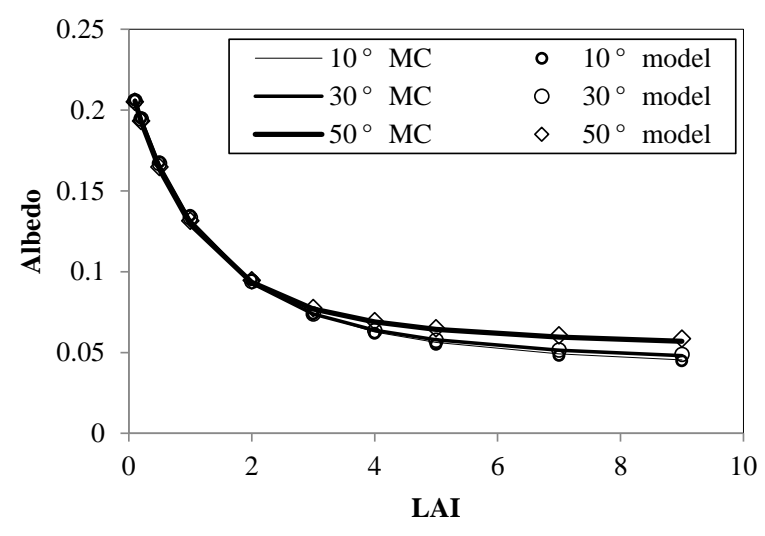

crop albedo (model against MC)
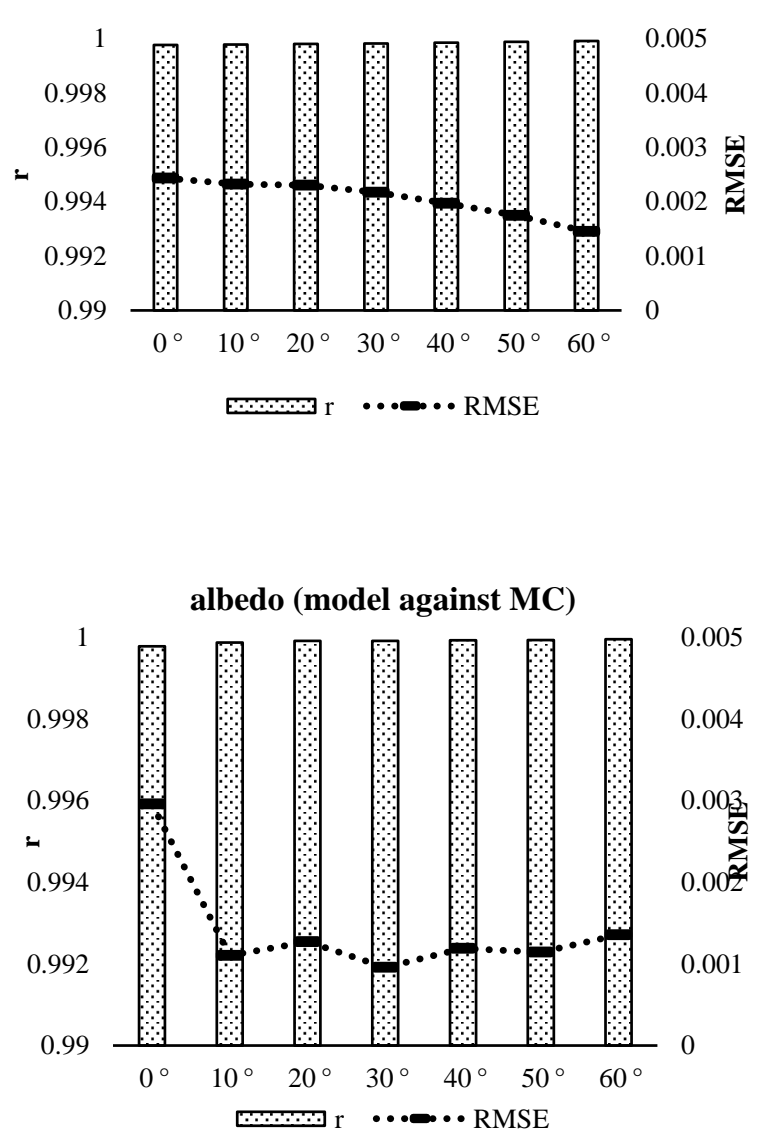

(b)

Figure 14. Relationships between LAI and crop albedo as predicted by the model (circles and diamonds) and MC (lines) under different solar zenith angles at the green band $\varphi=0^{\circ}$

(a) and $\varphi=90^{\circ}$ (b).

\subsection{Validation Using Ground Measurements}

The behavior of the albedo model developed in this study was also tested using measured parameters as input, and the result was compared to the measured albedo. The input parameters include the LAI, the fraction of diffuse radiation, the height and width of the row canopy, leaf reflectance/transmittance, soil albedo and the $\mathrm{G}$ function, which are obtained from field measurements. The albedo measurements include the spectral albedo and the shortwave albedo. The conversion from spectral albedo to broadband albedo has used the measured downward radiation as the weight, as detailed in Liang (2002).

\subsubsection{Site Description}

The study site at the Huailai Remote Sensing Station, which is located in Huailai, Hebei province, China $\left(40.349^{\circ} \mathrm{N}, 115.785^{\circ} \mathrm{E}\right)$, is one of the eco-hydrology and flux sites around China (Figure 15). The dominant crop of the Huailai Site is maize. The albedo model was tested using ground measurements of maize at two different growing stages. During the seedling stage, maize can be 
considered a non-continuous canopy, and until the canopy is closing, the maize can be considered a continuous canopy.

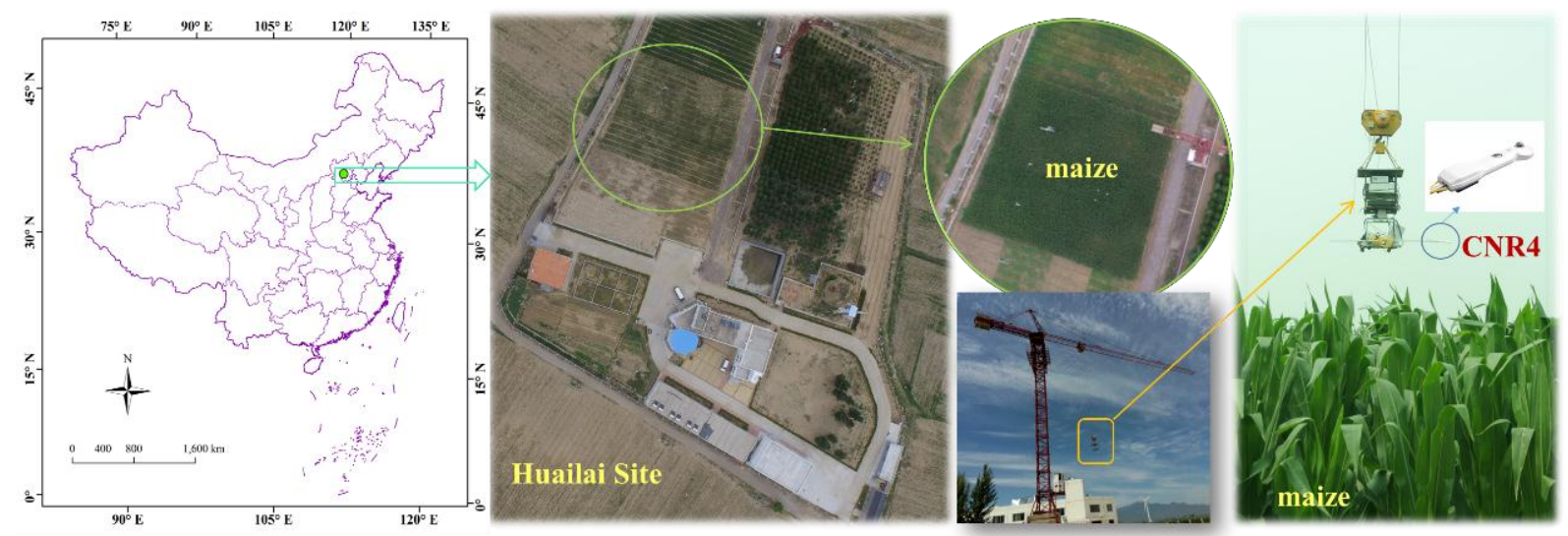

Figure 15. Position and aerial view photograph of the Huailai site.

\subsubsection{Measurements}

The albedo was collected at different periods of maize growth (Figure 16 and Table 2).
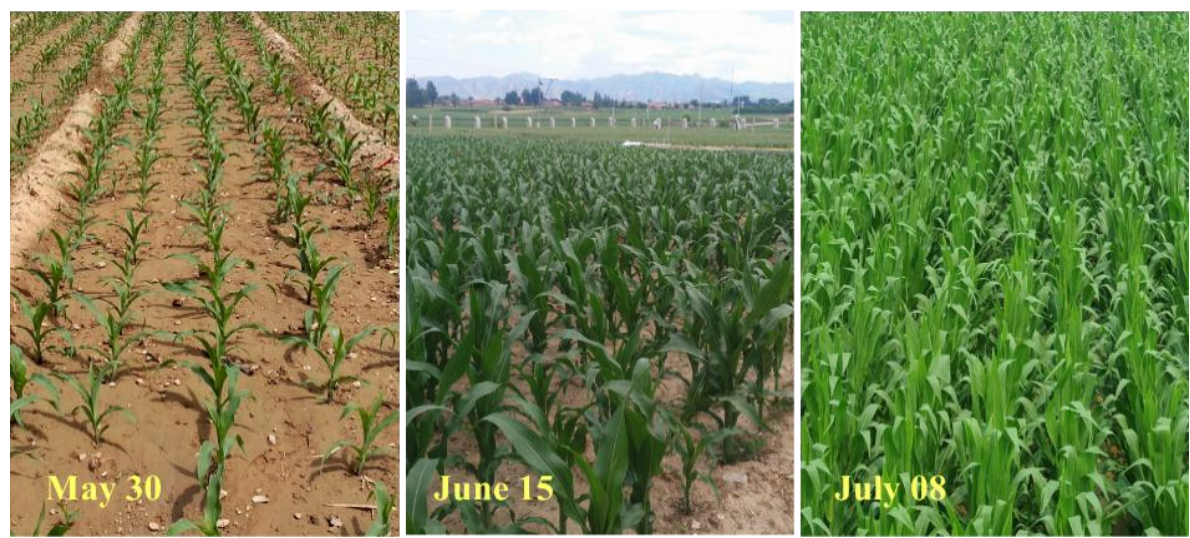

Figure 16. Position and aerial view photograph of the Huailai site.

(1) Spectral albedo of maize and soil: The spectral albedo was calculated as the ratio of the spectral downward to the upwelling radiation. The spectral surface radiosity was collected using an Analytical Spectral Devices (ASD) FieldSpec3 (FS3) spectroradiometer with the manufacturer's remote cosine receptor (RCR) foreoptic $\left(\sim 81^{\circ} \mathrm{HFOV}\right)$ held $0.5 \mathrm{~m}$ above the maize canopy (corresponding to a footprint of $3 \mathrm{~m}$ in radius. The diffuse incoming radiation is measured at the open terrain area with a black plane shading the direct incident radiation; the fraction of the diffuse radiation was calculated as the ratio of diffuse incoming radiation to downward radiation.

(2) Shortwave albedo: The shortwave albedo was derived from the upward/downward shortwave radiation, which was measured using a CNR4. The CNR4 was mounted on a pole and suspended from the platform of the tower crane, which allowed for position, height and level adjustment. The down-looking pyranometers looked down at the maize. Radiation was measured every 10 seconds and 
time averaged to one-minute intervals. The data logger clocks were synchronized to the second. Albedos were calculated as the ratio of reflected to incident radiation.

(3) $G$ function: $G$ is the mean projection of the leaf area unit in a plane perpendicular to the sunrays (Jonckheere I, 2004); G was estimated through the measurement of the probability density of the leaf angle distribution of the observed maize, which is the distribution of the leaf normals with respect to the upper hemisphere and was measured using a protractor. According to the statistical analysis of Ross (1981), the G function was simulated through the weighted average leaf angle of the observed maize (Figure 17).

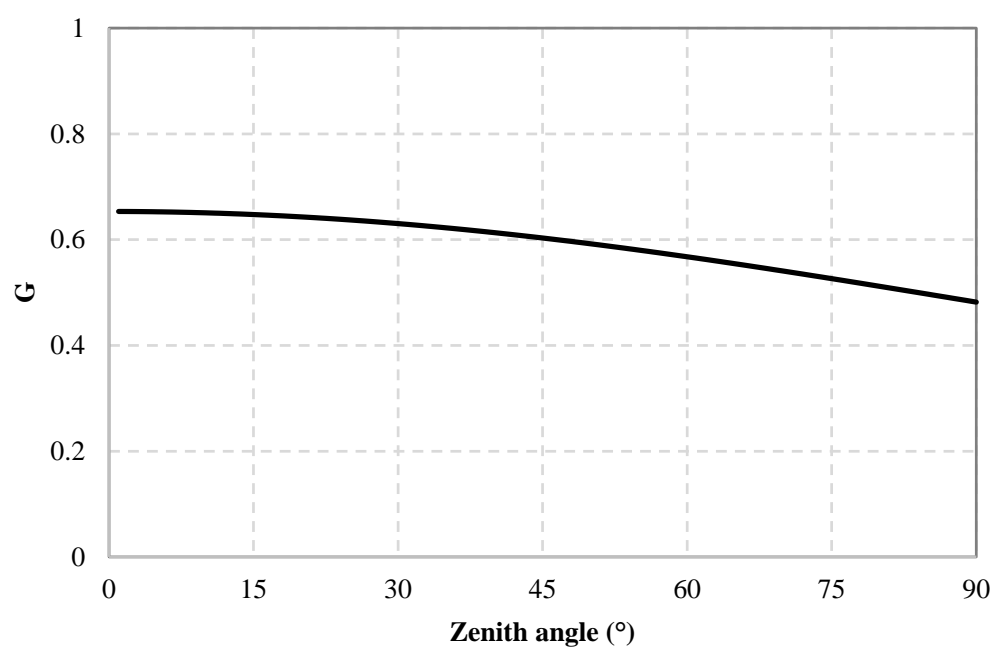

Figure 17. Relationship of the $\mathrm{G}$ function value with the zenith angle.

(4) Leaf reflectance/transmittance was measured using the Analytical Spectral Devices (ASD) FieldSpec3 (FS3) spectroradiometer with a leaf clip (Figure 18). The spectral measurement was averaged from three leaves, which were sampled from the top, middle and bottom of the plant.

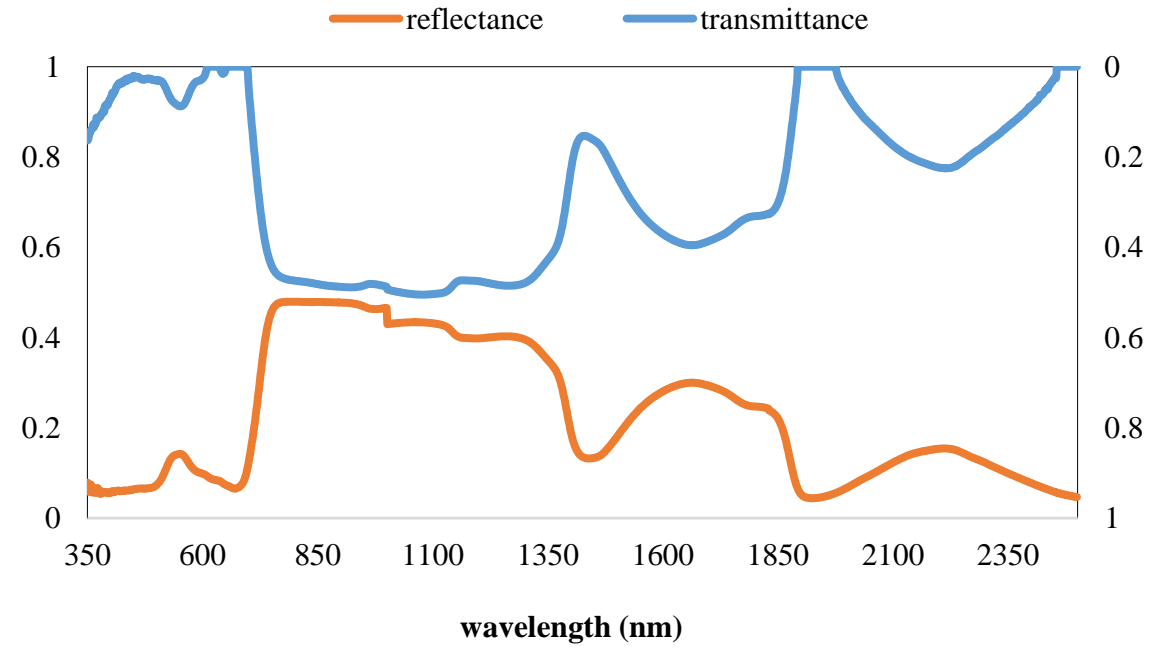

Figure 18. The measured reflectance and transmittance of maize leaves.

(5) The LAI was measured by using a destructive sampling method within a $5 \mathrm{~m} \times 5 \mathrm{~m}$ quadrant that belongs to the harvesting method. The LAI was accurately calculated using a CI-202.

(6) The width of one crop period was measured at the early period of the maize as the distance between two plants side-by-side between two rows. 
(7) The sun zenith angle values were the actual ones at the location of the measurements.

Table 2. The measured parameters on three individual dates.

\begin{tabular}{ccccccccc}
\hline Date & Time & LAI & $\begin{array}{c}\text { Solar } \\
\text { Zenith } \\
\text { Angle }\left(^{\circ}\right)\end{array}$ & $\begin{array}{c}\text { Solar } \\
\text { Azimuth } \\
\text { Angle }\left(^{\circ}\right)\end{array}$ & $\begin{array}{c}\text { Row } \\
\text { Width } \\
(\mathbf{m})\end{array}$ & $\begin{array}{c}\text { Gap } \\
\text { Width } \\
(\mathbf{m})\end{array}$ & $\begin{array}{c}\text { Canopy } \\
\text { Height }(\mathbf{m})\end{array}$ & $\begin{array}{c}\text { CNR4 } \\
\text { Measured } \\
\text { Albedo }\end{array}$ \\
\hline 30 May 2015 & $9: 36$ & 0.14 & 38.26 & 252.92 & 0.16 & 0.32 & 0.283 & 0.172 \\
14 June 2015 & $13: 48$ & 0.80 & 25.20 & 126.08 & 0.38 & 0.10 & 0.46 & 0.239 \\
8 July 2014 & $13: 10$ & 3.18 & 20.54 & 146.70 & -- & -- & 2.184 & 0.245 \\
\hline
\end{tabular}

\subsubsection{Comparison Results}

The model-simulated spectral albedo of the maize canopy agreed well with the ground-based spectral albedo (Figure 19). The spectral variation of the maize canopy can be accurately simulated using the proposed model for 30 May 2015. During this period, the maize was at the seedling stage, so the soil reflectance has a significant influence on the canopy albedo, which represents the situation on which the model focuses. The RMSE between the measured and modeled spectral albedo is 0.007 .

However, there is an obvious discrepancy between the calculated spectral albedo and the measured spectral albedo for 15 June 2015. Referring to the comparison result (Table 3) between the broadband albedo, it also proved that the calculated result has overestimated the canopy albedo. Given the strong correspondence between the calculated albedo and the ASD spectroradiometer albedo, we suspect that the discrepancy was caused by the overestimation of soil albedo in calculating the albedo. The soil albedo was measured from the adjacent bare farmland in which the soil was quite dry, while the maize was just irrigated and the underground soil was wetter. The larger soil moisture corresponds to lower soil reflectance.

None of the spectral albedo of the maize canopy was collected on 8 July 2015, as the canopy height was above $2 \mathrm{~m}$, which exceeded the limit that the handheld 1-m fiber probe could reach, so the calculated albedo for 8 July 2015 was directly validated using the broadband albedo measured with the CNR4. The calculated albedo was overestimated by approximately 0.004 , which is $1.63 \%$. During this period, the closing of the crop allowed the maize to be treated as continuous vegetation.

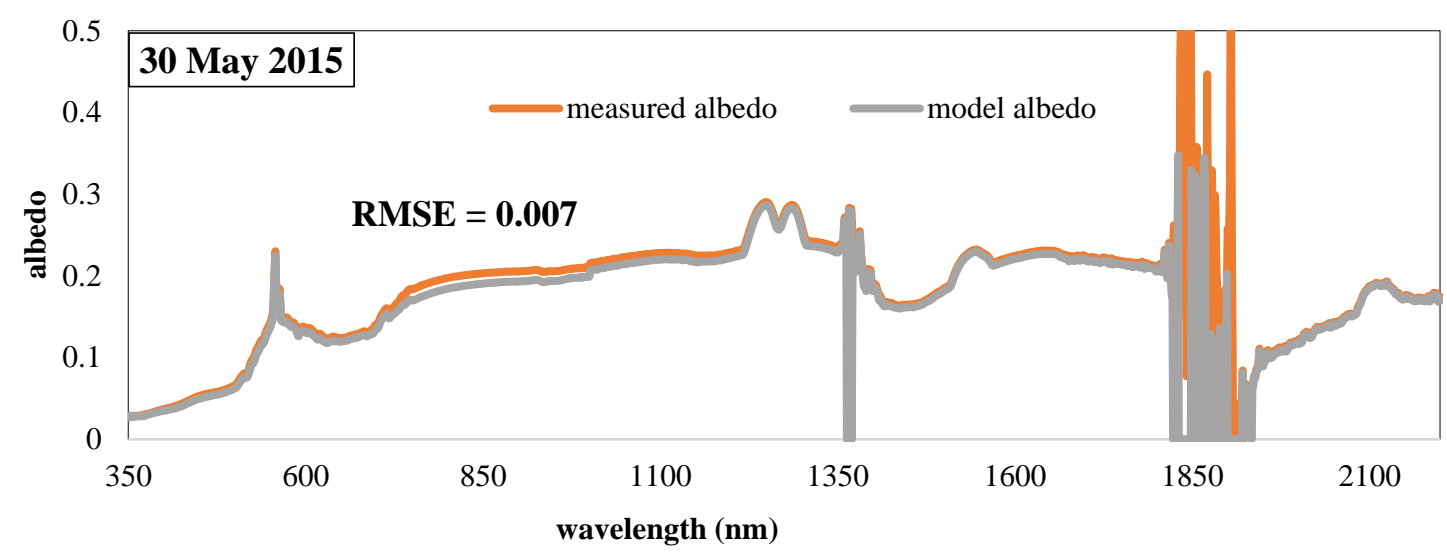

(a)

Figure 19. Cont. 


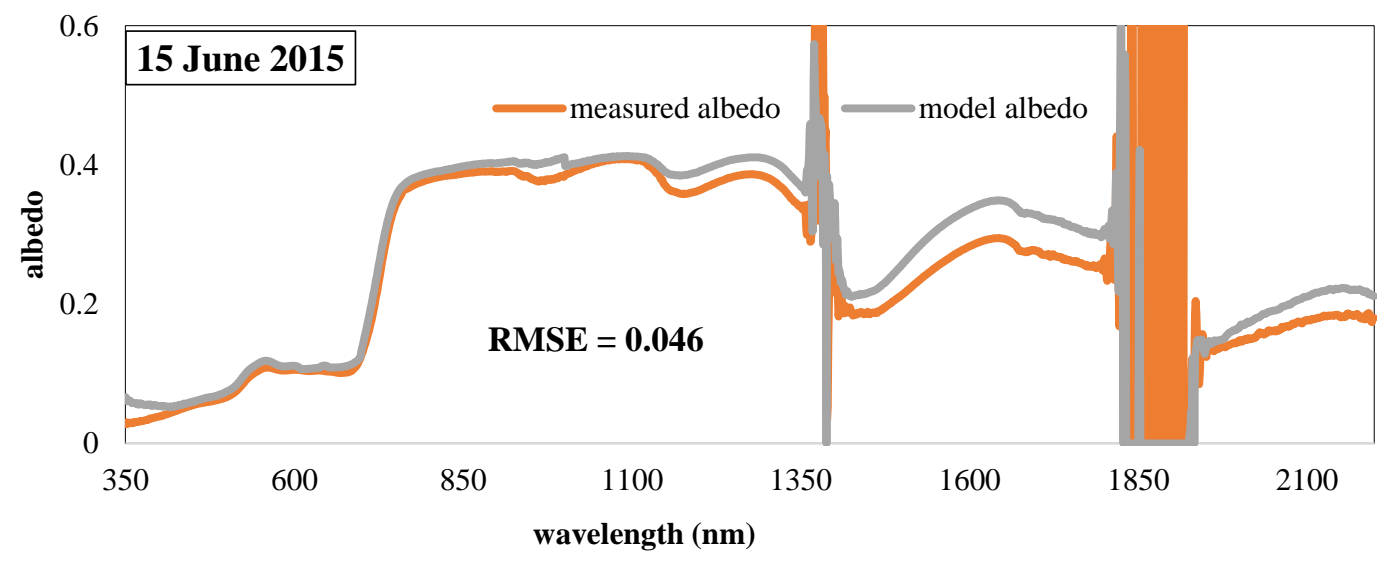

(b)

Figure 19. Comparison between the measured spectral albedo and model-simulated albedo on (a) 30 May and (b) 15 June.

From the comparison of the spectral albedo collected on 30 May 2015 and 15 June 2015, it has been proven that the albedo of agricultural surfaces changes considerably at different stages of the growing season. In the earlier stage, the canopy albedo is mainly determined by the optical characteristics of the soil particles, such as the moisture content of the topsoil. As the crop grows, the dominancy of the physical condition of the leaves and the structure of the crop becomes pronounced.

Table 3. Comparison between the broadband albedo directly measured using the CNR4 from the Analytical Spectral Devices (ASD)-measured and model-simulated spectral albedo.

\begin{tabular}{cccc}
\hline Date & CNR4 Measured Albedo & ASD Broadband Albedo & Model Broadband Albedo \\
\hline 30 May 2015 & 0.177 & 0.177 & 0.172 \\
15 June 2015 & 0.239 & 0.230 & 0.259 \\
8 July 2014 & 0.245 & -- & 0.249 \\
\hline
\end{tabular}

\section{Conclusions}

In this research, a physical model depicting crop albedo was derived from a forest albedo model. The model calculates albedo as a complement of canopy and soil absorptance. It considers multiple scatterings, both within the canopy and between the canopy and the soil, as well as diffuse sky radiation.

The model can be extended to row crops and discrete vegetation canopies using effective LAI instead of LAI. It has been proven that the new model is reliable and effective at acquiring vegetation albedo by comparing the results of the model and those of MC simulations. The model can typically be used in a simplified form, which builds a basis for the model to be used for remote sensing data in the future. Ground validation of the model showed that the discrepancy between the model-simulated albedo and the field measurements of continuous vegetation and crops is small in both spectral and broadband albedo.

As a physical model, it is more robust than empirical models in theory and would be more attractive for the assessment or monitoring of biophysical characteristics of vegetation. For an albedo diurnal course study, it would be a good choice to use this model, as the direct and diffuse irradiations are 
treated separately. Moreover, the model can be applied in vegetation albedo inversion from remote sensing data. These would be the next steps of the research.

\section{Acknowledgments}

This work was carried out with the National Natural Science Foundation of China (41501359, 41271346, 41571329, 41230747, 91425301), Postdoctoral Science Foundation of China (2015M570886), Open Fund of State Key Laboratory of Remote Sensing Science (OFSLRSS201518) and the Major State Basic Research Development Program of China (2013CB733402).

The authors thank the reviewers for their valuable and insightful comments and suggestions and the Elsevier language editing group for their contributions. We gratefully acknowledge the generously-shared LAI field measurements from Erli Cai and Baocheng Dou at Beijing Normal University. Special thanks are extended to Yelu Zeng of the Institute of Remote Sensing and Digital Earth for assisting in the soil albedo simulation, as well as to Biao Cao for cooperation in the field measurements.

\section{Author Contributions}

Jingjing Peng, Wenjie Fan and Xu Xiru conceived of and designed the experiments. Jingjing Peng, Lizhao Wang, Jvcai Li and Peng Zhao processed the data and performed the experiments. Qinhuo Liu, Wenjie Fan and Lizhao Wang participated in the discussion and analysis. Jingjing Peng wrote the whole manuscript. Wenjie Fan contributed to the conclusion and revision.

\section{Conflicts of Interest}

The authors declare no conflict of interest.

\section{References}

1. Jacob, F.; Olioso, A. Derivation of diurnal courses of albedo and reflected solar irradiance from airborne POLDER data acquired near solar noon. J. Geophys. Res.: Atmos. 2005, doi:10.1029/2004JD004888.

2. Bastiaanssen, W.G.; Chandrapala, L. Water balance variability across Sri Lanka for assessing agricultural and environmental water use. Agric. Water Manag. 2003, 58, 171-192.

3. Ottlé, C.; Vidal-Madjar, D. Assimilation of soil moisture inferred from infrared remote sensing in a hydrological model over the HAPEX-MOBILHY region. J. Hydrol. 1994, 158, 241-264.

4. Meher-Homji, V.M. Probable impact of deforestation on hydrological processes. In Tropical Forests and Climate; Springer: Dordrecht, The Netherlands, 1992; pp 163-173.

5. Ridgwell, A.; Singarayer, J.S.; Hetherington, A.M.; Valdes, P.J. Tackling regional climate change by leaf albedo bio-geoengineering. Curr. Biol. 2009, 19, 146-150.

6. Singarayer, J.S.; Ridgwell, A.; Irvine, P. Assessing the benefits of crop albedo bio-geoengineering. Environ. Res. Lett. 2009, 4, 45110.

7. Wang, Z.; Zeng, X.; Barlage, M.; Dickinson, R.E.; Gao, F.; Schaaf, C.B. Using MODIS BRDF and albedo data to evaluate global model land surface albedo. J. Hydrometeorol. 2004, 5, 3-14. 
8. Rechid, D.; Raddatz, T.J.; Jacob, D. Parameterization of snow-free land surface albedo as a function of vegetation phenology based on MODIS data and applied in climate modelling. Theor. Appl. Climatol. 2009, 95, 245-255.

9. Bonan, G.B.; Oleson, K.W.; Vertenstein, M.; Levis, S.; Zeng, X.; Dai, Y.; Dickinson, R.E.; Yang, Z. The land surface climatology of the community land model coupled to the NCAR community climate model. J. Climate 2002, 15, 3123-3149.

10. Liang, S. Quantitative Remote Sensing of Land Surfaces; John Wiley \& Sons: Hoboken, NJ, USA, 2005.

11. Ni, W.; Li, X. A coupled vegetation-soil bidirectional reflectance model for a semiarid landscape. Remote Sens. Environ. 2000, 74, 113-124.

12. Ni, W.; Woodcock, C.E. Effect of canopy structure and the presence of snow on the albedo of boreal conifer forests. J. Geophys. Res. 2000, 105, 11879-11888.

13. Schaaf, C.B.; Gao, F.; Strahler, A.H.; Lucht, W.; Li, X.; Tsang, T.; Strugnell, N.C.; Zhang, X.; Jin, Y.; Muller, J.; et al. First operational BRDF, albedo nadir reflectance products from MODIS. Remote Sens. Environ. 2002, 83, 135-148.

14. Roujean, J.L.; Leroy, M.; Deschamps, P.Y. A bidirectional reflectance model of the Earth's surface for the correction of remote sensing data. J. Geophys. Res.: Atmos. 1992, 97, 20455-20468.

15. White, H.P. Investigations of Boreal Forest Bidirectional Reflectance Factor (BRF). Ph.D. Degree, York University, Toronto, ON, Canada, 1999.

16. Shuai, Y.; Masek, J.G.; Gao, F.; Schaaf, C.B. An algorithm for the retrieval of 30-m snow-free albedo from Landsat surface reflectance and MODIS BRDF. Remote Sens. Environ. 2011, 115, 2204-2216.

17. North, P.R. Three-dimensional forest light interaction model using a Monte Carlo method. IEEE Trans. Geosci. Remote Sens. 1996, 34, 946-956.

18. Zhang, B.; Fan, W.; Xu, X.; Liu, Y. FAPAR and BRDF simulation for row crop using Monte Carlo method. In Proceedings of the IEEE International Geoscience and Remote Sensing Symposium (IGARSS), Quebec City, QC, Canada, 13-18 July 2014; pp. 812-815.

19. Disney, M.; Lewis, P. Spectral invariant behaviour of a complex 3D forest canopy. In Proceedings of the 10th ISPMSRS meeting, Davos, Switzerland, 12-14 March 2007; pp.1-6.

20. Rautiainen, M.; Stenberg, P. Application of photon recollision probability in coniferous canopy reflectance simulations. Remote Sens. Environ. 2005, 96, 98-107.

21. Mõttus, M. Photon recollision probability in discrete crown canopies. Remote Sens. Environ. 2007, 110, 176-185.

22. Manninen, T.; Stenberg, P. Simulation of the effect of snow covered forest floor on the total forest albedo. Agric. Forest Meteorol. 2009, 149, 303-319.

23. Stenberg, P.; Mottus, M.; Rautiainen, M. Modeling the spectral signature of forests: Application of remote sensing models to coniferous canopies. In Advances in Land Remote Sensing, Springer: Dordrecht, The Netherlands, 2008; pp 147-171.

24. Stenberg, P.; Lukeš, P.; Rautiainen, M.; Manninen, T. A new approach for simulating forest albedo based on spectral invariants. Remote Sens. Environ. 2013, 137, 12-16. 
25. Román, M.O.; Schaaf, C.B.; Lewis, P.; Gao, F.; Anderson, G.P.; Privette, J.L.; Strahler, A.H.; Woodcock, C.E.; Barnsley, M. Assessing the coupling between surface albedo derived from MODIS and the fraction of diffuse skylight over spatially-characterized landscapes. Remote Sens. Environ. 2010, 114, 738-760.

26. Fan, W.; Liu, Y.; Xu, X.; Chen, G.; Zhang, B. A new FAPAR analytical model based on the law of energy conservation: A case study in China. IEEE J. Sel. Top. Appl. Earth Obs. Remote Sens. 2014, 7, 3945-3955.

27. Hapke, B. Bidirectional reflectance spectroscopy: 1. Theory. J. Geophys. Res.: Solid Earth 1981, 86, 3039-3054.

28. Stenberg, P. Simple analytical formula for calculating average photon recollision probability in vegetation canopies. Remote Sens. Environ. 2007, 109, 221-224.

29. Chen, J.M.; Menges, C.H.; Leblanc, S.G. Global mapping of foliage clumping index using multi-angular satellite data. Remote Sens. Environ. 2005, 97, 447-457.

30. Chen, J.M.; Cihlar, J. Plant canopy gap-size analysis theory for improving optical measurements of leaf-area index. Appl. Opt. 1995, 34, 6211-6222.

31. Nilson, T. A theoretical analysis of the frequency of gaps in plant stands. Agric. Meteorol. 1971, 8, $25-38$.

32. Chen, J.M.; Leblanc, S.G. A four-scale bidirectional reflectance model based on canopy architecture. IEEE Trans. Geosci. Remote Sens. 1997, 35, 1316-1337.

33. Yan, B.; Xu, X.; Fan, W. A unified canopy bidirectional reflectance (BRDF) model for row ceops. Sci. Chin. Earth Sci. 2012, 55, 824-836.

34. Jacobs, A.; Van Pul, W. Seasonal changes in the albedo of a maize crop during two seasons. Agric. Forest Meteorol. 1990, 49, 351-360.

35. Pinty, B.; Verstraete, M.M.; Dickinson, R.E. A physical model for predicting bidirectional reflectances over bare soil. Remote Sens. Environ. 1989, 27, 273-288.

36. Ross, J. The Radiation Regime and Architecture of Plant Stands; Springer Science \& Business Media: Berlin, Germany, 2012.

37. Govaerts, Y.M.; Verstraete, M.M. Raytran: A Monte Carlo ray-tracing model to compute light scattering in three-dimensional heterogeneous media. IEEE Trans. Geosci. Remote Sens. 1998, 36, 493-505.

38. Ross, J.K.; Marshak, A.L. Calculation of canopy bidirectional reflectance using the Monte Carlo method. Remote Sens. Environ. 1988, 24, 213-225.

(C) 2015 by the authors; licensee MDPI, Basel, Switzerland. This article is an open access article distributed under the terms and conditions of the Creative Commons Attribution license (http://creativecommons.org/licenses/by/4.0/). 